\title{
The health effects of vitamin D supplementation: evidence from human studies
}

\author{
Roger Bouillon $\mathbb{1}^{1 凶}{ }^{凶}$, Despoina Manousaki², Cliff Rosen $\mathbb{D}^{3}$, Katerina Trajanoska \\ Fernando Rivadeneira (i) ${ }^{5}$ and J. Brent Richards ${ }^{6,7}$
}

Abstract | Vitamin D supplementation can prevent and cure nutritional rickets in infants and children. Preclinical and observational data suggest that the vitamin D endocrine system has a wide spectrum of skeletal and extra-skeletal activities. There is consensus that severe vitamin $D$ deficiency (serum 25-hydroxyvitamin D (25OHD) concentration $<30 \mathrm{nmol} / \mathrm{l}$ ) should be corrected, whereas most guidelines recommend serum $25 \mathrm{OHD}$ concentrations of $>50 \mathrm{nmol} / \mathrm{l}$ for optimal bone health in older adults. However, the causal link between vitamin $D$ and many extra-skeletal outcomes remains unclear. The VITAL, ViDA and D2d randomized clinical trials (combined number of participants $>30,000$ ) indicated that vitamin D supplementation of vitamin D-replete adults (baseline serum $25 \mathrm{OHD}>50 \mathrm{nmol} / \mathrm{l}$ ) does not prevent cancer, cardiovascular events, falls or progression to type 2 diabetes mellitus. Post hoc analysis has suggested some extra-skeletal benefits for individuals with vitamin D deficiency. Over 60 Mendelian randomization studies, designed to minimize bias from confounding, have evaluated the consequences of lifelong genetically lowered serum $25 \mathrm{OHD}$ concentrations on various outcomes and most studies have found null effects. Four Mendelian randomization studies found an increased risk of multiple sclerosis in individuals with genetically lowered serum 25OHD concentrations. In conclusion, supplementation of vitamin D-replete individuals does not provide demonstrable health benefits. This conclusion does not contradict older guidelines that severe vitamin $D$ deficiency should be prevented or corrected.

There is consensus that daily intake of 400 IU of vitamin $\mathrm{D}$ can prevent nutritional rickets in infants and children ${ }^{1}$. However, the skeletal effects of vitamin D deficiency in adults and older adults (aged $>65$ years), and the potential extra-skeletal effects of vitamin $\mathrm{D}$ are more controversial. Some people consider that vitamin D supplementation is futile ${ }^{2}$. By contrast, others have suggested that the vitamin $\mathrm{D}$ intake requirement is much higher than currently achieved by the general population and that people should aim to achieve 25-hydroxyvitamin D (25OHD, the major marker of vitamin D status) concentrations similar to those found in certain tribes in equatorial Africa with a sun exposure lifestyle that might be similar to that of early humans ${ }^{3-7}$.

The potential extra-skeletal effects of the vitamin D endocrine system (which refers to vitamin D in its active form, its precursors and metabolites, and vitamin D receptor) are based on several arguments. For example, the vitamin D receptor (VDR) and CYP27B1 (the enzyme primarily responsible for producing the active form of vitamin $\mathrm{D}, 1,25$-dihydroxyvitamin $\mathrm{D}$ or $\left.1,25(\mathrm{OH})_{2} \mathrm{D}_{3}\right)$ are widely expressed, including in tissues that are not involved in calcium or phosphate transport (FIG. 1). In addition, $\sim 3 \%$ of the human and mouse genomes are under the direct or indirect control of $1,25(\mathrm{OH})_{2} \mathrm{D}_{3}\left(\mathrm{REFS}^{8,9}\right)$. Finally, many diseases and illnesses in humans are associated with a poor vitamin D status, as measured by low serum levels of 25OHD. Therefore, one of the major clinical questions in the field is whether poor vitamin $\mathrm{D}$ status plays a causal role in the diseases and conditions associated with low $25 \mathrm{OHD}$ levels, such as cancer, impaired muscle strength and falls, and immune, metabolic or cardiovascular diseases. Furthermore, if the link is causal, the threshold serum level of $25 \mathrm{OHD}$ below which the risk of these diseases is increased must be identified ${ }^{8}$.

Up to about a decade ago, there was tremendous uncertainty about vitamin D supplementation for the maintenance of adequate health levels. Large, randomized placebo-controlled trials with clinically 


\section{Key points}

- Vitamin D and calcium supplementation can cure nutritional rickets and can modestly decrease the risk of major fractures in older adults with poor vitamin $D$ status or calcium intake.

- Large supplementation trials recruiting vitamin D-replete adults (serum $25 \mathrm{OHD}$ concentration $>50 \mathrm{nmol} / \mathrm{l}$ ) have demonstrated no effects on the incidence of cancer, cardiovascular events or type 2 diabetes mellitus (T2DM) and no benefits in terms of bone density and the risk of falls.

- Post-hoc analysis of large supplementation trials has suggested that supplementation of individuals with vitamin $\mathrm{D}$ deficiency modestly delays age-related bone loss and progression to T2DM, and improves lung function.

- A meta-analysis suggested that vitamin $\mathrm{D}$ supplementation results in a modest decrease in cancer mortality.

- Over 60 Mendelian randomization studies have examined causal links between genetically lower vitamin D levels and health outcomes; most studies generated null effects except four studies that demonstrated an increased risk of multiple sclerosis.

- In conclusion, supplementation of vitamin D-replete individuals does not generate overall health benefits; however, correction of severe vitamin D deficiency remains essential. important outcomes and/or surrogates had yet to be performed and only a few meta-analyses of randomized controlled trials (RCTs) were available. On the other hand, many observational studies had been conducted that were confounded by multiple variables. The Institute of Medicine (IOM) guidelines were developed to provide an answer based on the best available evidence at that time ${ }^{10}$. The IOM Committee established minimal dosing to maintain adequate serum levels of 25OHD in young and older individuals and established the tolerable upper limits of supplementation. The Committee also examined the totality of evidence relating vitamin $\mathrm{D}$ supplementation to numerous outcomes, and concluded that large randomized trials were needed to establish efficacy and safety. Other societies, such as the Endocrine Society ${ }^{11}$, and governmental organizations also generated a variety of guidelines ${ }^{12}$. The minimal serum level of $25 \mathrm{OHD}$ that was considered to provide vitamin D sufficiency varied from $30 \mathrm{nmol} / \mathrm{l}$ (UK Scientific Advisory Committee on Nutrition ${ }^{13}$ ), to $50 \mathrm{nmol} / \mathrm{l}$ (IOM and many other governmental guidelines) ${ }^{12,14}, 75 \mathrm{nmol} / \mathrm{l}$ (Endocrine Society and some other

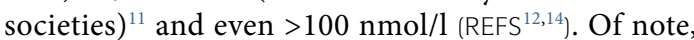
serum levels of $>100 \mathrm{nmol} / \mathrm{l}$ are found in people living in Africa under conditions of sun exposure supposed to be similar to that of early humans.

\footnotetext{
Author addresses

${ }^{1}$ Clinical and Experimental Endocrinology, Department of Chronic Diseases and Metabolism, KU Leuven, Leuven, Belgium.

${ }^{2}$ Research Center of the Sainte-Justine University Hospital, University of Montreal, Montreal, Quebec, Canada.

${ }^{3}$ Maine Medical Center Research Institute, Scarborough, ME, USA.

${ }^{4}$ Department of Internal Medicine, Erasmus MC University Medical Center, Rotterdam, Netherlands.

${ }^{5}$ Translational Skeletal Genomics, Department of Internal Medicine, Erasmus MC University Medical Center, Rotterdam, Netherlands.

${ }^{6}$ Centre for Clinical Epidemiology, Lady Davis Institute for Medical Research, Jewish General Hospital, Montreal, Quebec, Canada.

${ }^{7}$ Departments of Medicine, Human Genetics, Epidemiology and Biostatistics, McGill

University, Montreal, Quebec, Canada.
}

In this Review, we summarize the results of recent (2017-2020) RCTs as well as Mendelian randomization studies, while not reviewing observational studies, which have been well-documented previously ${ }^{9}$. We have chosen these two study designs because they are both types of causal inference studies and can help provide insights into the role of vitamin D in the aetiology of common diseases. The reviewed studies do not provide evidence that vitamin $\mathrm{D}$ supplementation prevents negative health outcomes in vitamin D-replete adults. However, all these studies reveal new suggestions for potential effects of vitamin D supplementation. Note that throughout the text vitamin $\mathrm{D}$ refers to vitamin $\mathrm{D}_{3}$ unless otherwise specified.

\section{RCTs: 2017-2020}

Many small-scale RCTs have been published over the past few years. In addition, several large studies have generated a wealth of new data (TABLE 1; Supplementary Box 1). The new major RCTs deal with more than 35,000 study participants who have a generally better health profile than participants in older studies. These studies used higher dosages than previous studies and the volunteers were mostly vitamin D-replete at baseline. These differences might explain why these large RCTs generated mostly null results in the intention-to-treat (ITT) analysis.

The largest trial to date is the VITAL study ${ }^{15}$ that recruited more than 25,000 adults from 44 centres in the USA and evaluated daily doses (2,000 IU) of vitamin D for a mean duration of 5.3 years. The Vitamin D Assessment Study (ViDA study) ${ }^{16}$ evaluated the effects of monthly high-dose vitamin D supplementation in more than 5,000 adults in New Zealand followed for a mean duration of 3.3 years. The primary aim of the D2d study of 2,423 US participants was to evaluate the effects of a daily dose of vitamin D (4,000 IU per day) for a mean duration of 2.5 years on the conversion of prediabetes to type 2 diabetes mellitus (T2DM) ${ }^{17}$. The DO-HEALTH study evaluated the effects of vitamin D (2,000 IU per day) in 2,157 older adults in Europe for a duration of 3 years ${ }^{18}$. The Calgary study was not really a megatrial, as it included only 311 Canadian adults and explored the effects on bone structure and quality and the safety of daily high-dose vitamin D (4,000 and 10,000 IU versus 400 IU) for 3 years ${ }^{18-20}$.

\section{Mendelian randomization studies}

Mendelian randomization is an established genetic epidemiological method, which can be used to test whether genetically decreased 25OHD levels are associated with increased risk of disease. To do this, Mendelian randomization uses single nucleotide polymorphisms (SNPs) that are associated with 25OHD levels in genome-wide association studies (GWAS) as instruments to infer 25OHD levels. Depending on their number, these SNPs can explain from $2 \%$ to $10 \%$ of the variance in $25 \mathrm{OHD}$ levels. This approach offers an alternative analytical technique able to reduce bias from confounding and reverse causation present in observational studies and re-estimates observations in a framework enabling causal inference (Supplementary Box 2). The very large number of Mendelian randomization studies 


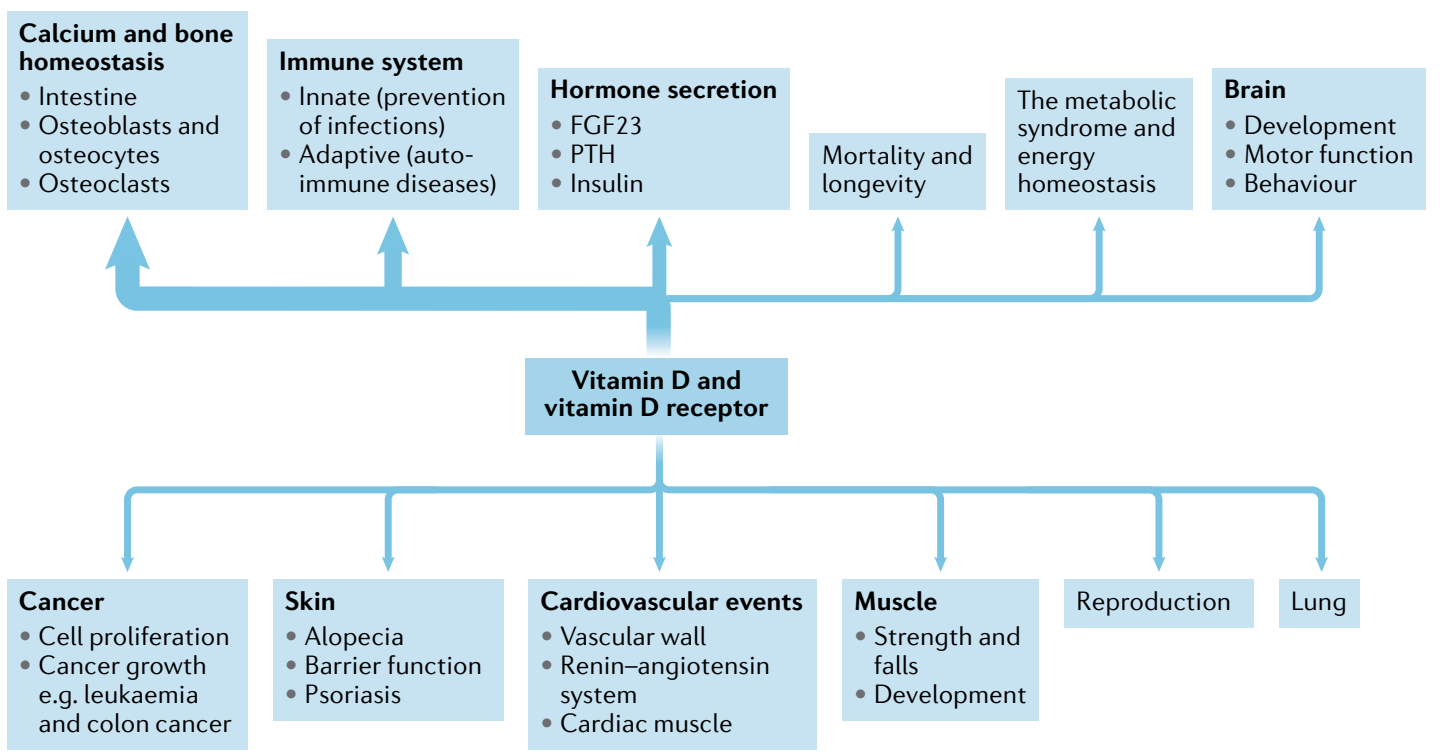

Fig. 1 | The many plausible target tissues and effects of the vitamin D endocrine system. The potential skeletal and extra-skeletal target tissues and effects of the vitamin D endocrine system (vitamin D and vitamin D receptor) as based on preclinical and observational studies, Mendelian randomization studies and randomized controlled trials (RCTs). In vitro studies have identified many molecular and genetic targets of vitamin D action. Animal models have confirmed a variety of skeletal and extra-skeletal actions. Human observational data are largely in line with preclinical data. However, Mendelian randomization studies and RCTs have not confirmed such a widespread action profile in vitamin D-replete adults. Therefore, the health consequences of poor vitamin $D$ status remain controversial. The strength of the relationship between the vitamin D endocrine system and health effects are indicated by the arrow thickness. FGF23, fibroblast growth factor 23; PTH, parathyroid hormone.

of vitamin D have also generated mostly null results; however, they have been handicapped by the low power to predict decreased serum $25 \mathrm{OHD}$ concentrations.

\section{Effects of vitamin D on health outcomes T2DM}

Many observational studies suggest a link between low vitamin $\mathrm{D}$ status and $\mathrm{T} 2 \mathrm{DM}^{9}$.

Evidence from RCTs. In the large D2d RCT of patients with prediabetes (TABLE 1), vitamin D supplementation only showed a non-significant trend to slow down the progression of prediabetes into T2DM. The study design intentionally included people with a high risk of progression to T2DM, who received vitamin D (4,000 IU per day). In the ITT analysis, the hazard ratio for the development of T2DM in the group receiving vitamin $\mathrm{D}$ was 0.88 (95\% CI 0.75-1.04; $P=0.12$ ) compared with the placebo group. In a post hoc analysis, however, a significant effect was observed in individuals with a baseline BMI below $30 \mathrm{mg} / \mathrm{m}^{2}$, severe vitamin $\mathrm{D}$ deficiency at baseline, perfect adherence to treatment during the study or serum $25 \mathrm{OHD}$ above $100 \mathrm{nmol} / \mathrm{l}$ throughout the study $^{21}$ (TABLES 2,3). Analysis of the combined data from the D2d trial and two other trials specifically designed and conducted to investigate the effectiveness of vitamin D supplementation in preventing T2DM showed that vitamin D supplementation (when compared with placebo) reduced the risk of developing T2DM from $23 \%$ to $13 \%$ (a $10 \%$ reduction) in persons with prediabetes not selected for vitamin D deficiency ${ }^{22}$. This finding is in line with two meta analyses published in 2020 dealing with eight ${ }^{23}$ and seven ${ }^{24}$ RCTs in people with prediabetes. These meta-analyses concluded that vitamin D supplementation decreased the risk to progress to T2DM by about $10 \%$, especially when using doses above 1,000 IU per day and in participants without obesity. Participant-level meta-analysis of these trials might provide a better estimate of risk reduction and identify populations of patients with prediabetes who are likely to benefit the most from vitamin $\mathrm{D}$ supplementation.

Evidence from Mendelian randomization. Since 2015, seven large Mendelian randomization studies have investigated the causal effect of genetically altered $25 \mathrm{OHD}$ levels on risk of T2DM and related traits (Supplementary Box 3). These Mendelian randomization studies included very large numbers of participants and mostly recruited white individuals and Chinese individuals. One study ${ }^{25}$ generated conflicting results, as part of the study using only two SNPs concluded that high predicted serum levels of 25OHD protected against T2DM (OR 0.86 of T2DM for a $25 \mathrm{nmol} / \mathrm{l}$ higher $25 \mathrm{OHD}$ concentration than that seen in the general population). However, in a slightly larger group of the same study that included two additional SNPs, the odds ratio became insignificant (Supplementary Box 3). All the other Mendelian randomization studies, including more than 500,000 volunteers, did not find a significant odds ratio for the relationship between predicted 25OHD and risk of T2DM.

Vitamin D and T2DM - summary. Although observational data have consistently confirmed lower serum $25 \mathrm{OHD}$ concentrations in patients with T2DM or 
Table 1 | Overview of the large vitamin D supplementation clinical trials 2017-2020

\begin{tabular}{|c|c|c|c|c|c|c|c|c|c|}
\hline \multirow[t]{2}{*}{ Study } & \multirow[t]{2}{*}{ Country } & \multirow{2}{*}{$\begin{array}{l}\text { Number of } \\
\text { patients }\end{array}$} & \multirow{2}{*}{$\begin{array}{l}\text { Age } \\
\text { (years, } \\
\text { mean } \pm S D \text { ) }\end{array}$} & \multirow{2}{*}{$\begin{array}{l}\text { Ethnicity } \\
\text { (\% white } \\
\text { ethnicity) }\end{array}$} & \multicolumn{2}{|c|}{ Serum $250 H D(\mathrm{ng} / \mathrm{ml})$} & \multirow{2}{*}{$\begin{array}{l}\text { Duration of } \\
\text { follow-up } \\
\text { (years) }\end{array}$} & \multirow{2}{*}{$\begin{array}{l}\text { Intervention } \\
\text { (vitamin D } \\
\text { vs placebo) }\end{array}$} & \multirow{2}{*}{$\begin{array}{l}\text { Primary } \\
\text { outcome(s) }\end{array}$} \\
\hline & & & & & Baseline & Final $^{b}$ & & & \\
\hline VITAL ${ }^{c}$ & USA & 25,874 & $67 \pm 7$ & 71 & $30.8 \pm 10$ & $42 \pm 10$ & 5.3 & 2,000 IU per day & $\begin{array}{l}\text { Cancer and } \\
\text { cardiovascular } \\
\text { disease }\end{array}$ \\
\hline ViDA & $\begin{array}{l}\text { New } \\
\text { Zealand }\end{array}$ & 5,110 & $66 \pm 8$ & 83 & $26.5 \pm 9^{d}$ & $54 \pm 16$ & 3.3 & $\begin{array}{l}\text { One dose of } 200,000 \\
\text { IU and } 100,000 I U \\
\text { per month }\end{array}$ & $\begin{array}{l}\text { Cardiovascular } \\
\text { events and } \\
\text { mortality }\end{array}$ \\
\hline DO-HEALTH & Europe & 2,157 & $74.9 \pm 4.4$ & NM & $22.4 \pm 8.4$ & $37.6 \pm 11.3$ & 3 & 2,000 IU per daye & $\begin{array}{l}\text { Six health } \\
\text { outcomes }^{f}\end{array}$ \\
\hline Calgary & Canada & 373 & $62 \pm 4$ & 94 & $31 \pm 8$ & $80 \pm 16^{9}$ & 3 & $\begin{array}{l}400,4,000 \text { or } 10,000 \\
\text { IU per day }\end{array}$ & BMD \\
\hline
\end{tabular}

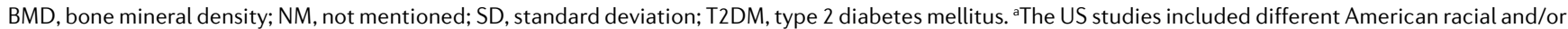
ethnic groups including Black people and Hispanic people. The ViDA study included Asian people and a small number of indigenous Māori individuals. ${ }^{b}$ Final serum concentration of $25 \mathrm{OHD}$ in the vitamin D-treated groups only. ${ }^{\circ}$ The VITAL trial is in fact a two-by-two factorial design study evaluating the potential benefits of vitamin $D$ and marine $n-3$ fatty acids ( $1 \mathrm{~g}$ per day). ${ }^{\mathrm{d} D e-s e a s o n a l i z e d ~ m e a n ~ v a l u e s . ~}{ }^{\mathrm{e}} \mathrm{A} 2 \times 2 \times 2$ factorial design evaluating vitamin $\mathrm{D}$, $\mathrm{n}-3$ fatty acids and exercise. ${ }^{\mathrm{f} S y s}$ tolic and diastolic blood pressure, physical and cognitive performance, non-vertebral fractures and infections. ${ }^{9} \mathrm{Highest}$ value in the $10,000 \mathrm{IU}$ per day group at 18 months.

the metabolic syndrome ${ }^{9}$, most Mendelian randomization studies have not supported these conclusions. Importantly, the large D2d RCT only showed a non-significant trend to slow down the progression of prediabetes into T2DM. In a small subgroup of individuals with overweight (rather than obesity) and prediabetes, supplementation provided some modest benefit, albeit lower than lifestyle modifications or metformin ${ }^{26}$. Furthermore, analysis of the combined results of the D2d trial and two other trials showed that vitamin D supplementation reduced the risk of developing T2DM in people with prediabetes not selected for vitamin $\mathrm{D}$ deficiency ${ }^{22}$. Additional studies or more in-depth analysis of the existing studies are needed to validate these findings. In summary, the evidence from large-scale Mendelian randomization studies and RCTs are convergent and do not support the use of vitamin D supplementation for the prevention of T2DM.

\section{Cancer}

Strong preclinical data exist that link vitamin $\mathrm{D}$ with cell cycle control and cancer. Furthermore, many observational studies have associated poor vitamin D status with increased risk of cancer or poor prognosis ${ }^{27}$.

Evidence from RCTs. The largest RCT (VITAL) did not find an effect of daily vitamin D supplementation on invasive cancer incidence (HR 0.96, 95\% CI 0.88-1.06) in US adults during a 5.3-year follow-up ${ }^{15}$. Further subanalysis (not statistically corrected for multiple comparisons) revealed a significant reduction in cancer risk in individuals with a normal BMI $\left(<25 \mathrm{~kg} / \mathrm{m}^{2}\right)$ and a trend for decreased cancer risk in African Americans. Baseline serum $25 \mathrm{OHD}$ concentrations did not influence cancer incidence or mortality but the number of participants with vitamin $\mathrm{D}$ deficiency at baseline $(<50 \mathrm{nmol} / \mathrm{l})$ was low $(\sim 10 \% \text { of the total cohort })^{28}$. In the ViDA trial in New Zealand adults, monthly vitamin D supplementation did not modify cancer incidence (overall or specific types of cancer, excluding non-melanoma skin cancers) with an overall hazard ratio of 1.01 (95\% CI $0.81-1.25)^{16}$.
Cancer mortality, as evaluated in a Cochrane systematic review ${ }^{29}$, was modestly decreased by vitamin $\mathrm{D}$ supplementation in four RCTs (44,492 participants), with a relative risk (RR) for cancer mortality of 0.88 (95\% CI $0.78-0.98$ ) in individuals receiving a mean daily dose of 1,146 IU (compared with no supplementation) during a mean follow-up of 6.3 years. Cancer mortality was also evaluated in several large RCTs (Supplementary Box 4). In the ITT analysis of the VITAL trial, a non-significant trend of reduction in total cancer mortality (HR 0.83 , 95\% CI 0.67-1.02) was observed in the vitamin D supplementation group. When excluding cancer deaths during the first year, or the first and second year after randomization, a significant reduction in cancer mortality was observed in the vitamin D supplementation group compared with no supplementation (HR 0.75, 95\% CI 0.59-0.96). In a Kaplan-Meier plot, the cumulative increased risk of cancer mortality was visible from year 4 of follow-up onwards ${ }^{28}$. In the ViDA trial, however, the number of cancer deaths was not influenced by vitamin D supplementation (HR 0.97), even after exclusion of cancer deaths registered in the first year after randomization (HR 0.95$)^{16}$. This discrepancy might be related to the short duration of follow-up. The ViDA trial lasted $<4$ years, whereas the effect of vitamin D supplementation in the VITAL study was only significant 4 years after randomization. An updated summary from the VITAL study ${ }^{28}$ confirmed a small but significant effect on cancer death in vitamin $\mathrm{D}$-supplemented individuals (HR 0.87, 95\% CI 0.79-0.96; $P=0.005$ ). As the final serum concentration of 25OHD in the VITAL trial $(\sim 110 \mathrm{nmol} / \mathrm{l})$ and the ViDA trial $(\sim 125 \mathrm{nmol} / \mathrm{l}$ or $50 \mathrm{ng} / \mathrm{ml})$ were in the high normal range it is unlikely that higher doses would be more effective.

Evidence from Mendelian randomization. The Ovarian Cancer Association Consortium (10,065 patients with ovarian cancer, 21,654 control individuals ${ }^{30}$ found a $27 \%$ increase in the risk of epithelial ovarian cancer per $20 \mathrm{nmol} / \mathrm{l}$ decrease in genetically determined 25OHD serum concentration (OR 1.27, 95\% CI 1.06-1.51). 
However, the results were not corroborated by another Mendelian randomization study ${ }^{31}$ which also showed no evidence of an association between 25OHD and risk of colorectal, breast, prostate, lung and pancreatic cancer or neuroblastoma. Similar findings were reported in a separate study ${ }^{32}$ in relation to total incident cancer and cancer subtypes such as breast, colorectal and lung cancer in 23,294 women. A null effect of genetically determined 25OHD on colorectal carcinoma was confirmed in men and women after including two additional $\mathrm{SNPs}^{33}$. Similarly, a large-scale two-sample Mendelian randomization study (122,977 patients with breast cancer and 79,148 patients with prostate cancer) did not show any effects of genetically predicted 25OHD concentrations on these cancers (Supplementary Box 5). Evidence from Mendelian randomization also refutes a link between $25 \mathrm{OHD}$ concentrations with risk of oesophageal adenocarcinoma $^{34}$, melanoma and non-melanoma skin cancer $^{35}$ (Supplementary Box 5).

Vitamin D and cancer - summary. No effects of vitamin D supplementation on cancer risk were observed in the large VITAL and ViDA trials. In line with prior studies and Mendelian randomization results, it thus seems clear that vitamin $\mathrm{D}$ supplementation in vitamin D-replete adults does not change cancer risk. However, a subanalysis of the VITAL trial showed that vitamin D

\section{Table 2 | Potential extra-skeletal benefits of vitamin D supplementation} in individuals with vitamin $D$ deficiency at baseline

\begin{tabular}{|c|c|c|c|}
\hline $\begin{array}{l}\text { Authors } \\
\text { and ref. }\end{array}$ & Outcome & Overall results & $\begin{array}{l}\text { Results in vitamin } \\
\text { D-deficient } \\
\text { participants }\end{array}$ \\
\hline \multirow{4}{*}{$\begin{array}{l}\text { Sluyter } \\
\text { et al. } .^{4}\end{array}$} & Brachial blood pressure & NS & NS \\
\hline & $\begin{array}{l}\text { Central blood pressure: } \\
\text { systolic }\end{array}$ & NS & $-7.5 \mathrm{~mm}(P=0.03)$ \\
\hline & $\begin{array}{l}\text { Central blood pressure: } \\
\text { diastolic }\end{array}$ & NS & NS \\
\hline & Six other parameters & NS & $P=0.03-0.003$ \\
\hline \multirow[t]{4}{*}{$\begin{array}{l}\text { Sluyter } \\
\text { et al. }{ }^{86}\end{array}$} & $\begin{array}{l}\text { All participants: lung function } \\
\text { (FEV1) }\end{array}$ & $+16 \mathrm{ml}(\mathrm{NS})$ & +39 ml (NS) \\
\hline & $\begin{array}{l}\text { Substudy ( } n=442, \text { follow-up } \\
1.1 \text { years): participants with } \\
\text { asthma or COPD }\end{array}$ & $+40 \mathrm{ml}(\mathrm{NS})$ & $+109 \mathrm{ml}(P=0.08)$ \\
\hline & $\begin{array}{l}\text { Substudy ( } n=442 \text {, follow-up } \\
1.1 \text { years): participants who } \\
\text { had ever-smoked }\end{array}$ & $+57 \mathrm{ml}(P=0.03)$ & $+112 \mathrm{ml}(P=0.04)$ \\
\hline & $\begin{array}{l}\text { Substudy ( } n=442, \text { follow-up } \\
1.1 \text { years): participants who } \\
\text { had ever-smoked with asthma } \\
\text { or COPD }\end{array}$ & $+160 \mathrm{ml}(P=0.004)$ & NA \\
\hline \multirow{3}{*}{$\begin{array}{l}\text { Wu et al. } \\
\left(\text { REF. }^{124}\right)\end{array}$} & Pain impact score & NS & NS \\
\hline & Prescription of opioids & NS & NS \\
\hline & Prescription of NSAIDs & NS & RR $0.87(P=0.01)$ \\
\hline $\begin{array}{l}\text { Pittas } \\
\text { et al. }^{\text {b }} \\
\text { (REF. }^{17} \text { ) }\end{array}$ & $\begin{array}{l}\text { Progression of prediabetes } \\
\text { into T2DM: post hoc analysis } \\
(n=103)\end{array}$ & NS & $\begin{array}{l}\mathrm{HR} 0.38(95 \% \mathrm{Cl} \\
0.18-0.80)\end{array}$ \\
\hline
\end{tabular}

Findings are from post hoc analysis or subgroup analysis of the large clinical trials. COPD, chronic obstructive pulmonary disease; FEV1, forced expiratory volume in $1 \mathrm{~s}$; NA, not applicable; NS, not significant; RR, relative risk; T2DM, type 2 diabetes mellitus. ${ }^{a}$ Serum $25 \mathrm{OHD}<50 \mathrm{nmol} / \mathrm{l}$. bSerum $25 \mathrm{OHD}<30 \mathrm{nmol} / \mathrm{l}$. supplementation might have some minor benefits in individuals with a normal BMI, but this finding was not corrected for multiple end point analysis ${ }^{15}$. In addition, several independent trials have suggested, in post hoc analysis, potential benefits of vitamin D supplementation on cancer mortality, especially when the follow-up is longer than 4 years $^{28}$ (Supplementary Box 4). Therefore, a link between vitamin D status and cancer incidence or mortality cannot be excluded, but will be very difficult to verify. Small changes in vitamin D status are unlikely to affect cancer incidence based on several Mendelian randomization studies.

\section{Cardiovascular events}

Major cardiovascular events. The results of any observational studies in humans are in line with preclinical data and have demonstrated a consistent association between low vitamin D status and increased risk of cardiovascular diseases, hypertension and cardiovascular events, including ischaemic cardiac events, cardiomyopathy, congestive heart failure, stroke and even cardiovascular mortality. In a meta-analysis of nearly 850,000 individuals, low serum $25 \mathrm{OHD}$ concentrations were associated with an increased risk of cardiovascular events (RR 1.43, comparing individuals with the lowest vitamin D status with individuals with a better vitamin D status) ${ }^{36}$.

Two large RCTs (VITAL and ViDA) were designed to include cardiovascular events as one of their primary end points ${ }^{15,37}$. During the 5.3 years of follow-up in the VITAL trial, the hazard ratio for the expanded composite end point of major cardiovascular events including coronary revascularization was 0.97 (95\% CI 0.85-1.12) in the vitamin D supplementation group, compared with placebo. A similar hazard ratio was found for cardiovascular death (HR 1.11, 95\% CI 0.88-1.40), or death from any cause. Exclusion of cardiovascular events or deaths during the first 2 years of follow-up did not change the overall results. Similarly, in the ViDA study, the primary outcome of major cardiovascular events was not influenced by monthly vitamin D supplementation over 3.3 years ${ }^{37}$. The adjusted hazard ratio for a combination of major cardiovascular events in the vitamin D supplementation group was 1.02 (95\% CI 0.87-1.20) compared with placebo, and such null findings also applied for a large list of secondary end points (myocardial infarction, heart failure, stroke and hypertension, among others), or cardiovascular deaths. Findings were not dependent on the baseline serum 25OHD concentration or previous cardiovascular status. When the results of these two major trials (including together more than 30,000 participants) were combined with those of previous studies evaluating the potential effects of vitamin D supplementation, a similar general conclusion of no effect of vitamin $\mathrm{D}$ supplementation was reached. An analysis of 21 RCTs including more than 80,000 participants showed that major cardiovascular events were not influenced by vitamin D supplementation ${ }^{38}$. The hazard ratios for myocardial infarction, stroke or cardiovascular death were all close to 1 and the $95 \%$ confidence intervals included the null. The results are uniformly concordant despite variation in target groups, baseline vitamin D status and vitamin dosage or regimens. Furthermore, 
Table 3 | Potential extra-skeletal benefits of vitamin D supplementation on T2DM or cancer incidence

\begin{tabular}{|c|c|c|c|c|}
\hline Authors and ref. & $\begin{array}{l}\text { Subgroup of original study } \\
\text { population }\end{array}$ & Number of patients & Odds ratio & $\begin{array}{l}95 \% \text { confidence } \\
\text { interval }\end{array}$ \\
\hline \multicolumn{5}{|c|}{ Progression of prediabetes to T2DM } \\
\hline \multirow[t]{2}{*}{ Pittas et al. ${ }^{17}$} & Group with perfect adherence & NM & 0.84 & $0.71-1.00$ \\
\hline & Baseline $\mathrm{BMI}<30 \mathrm{~kg} / \mathrm{m}^{2}$ & 187 & 0.71 & $0.53-0.95$ \\
\hline \multirow[t]{2}{*}{ Dawson-Hughes et al. ${ }^{21}$} & $\begin{array}{l}\text { Serum level of } 25 \mathrm{OHD} \text { throughout } \\
\text { the study of } 40-50 \mathrm{ng} / \mathrm{ml}\end{array}$ & 319 & 0.48 & $0.29-0.80$ \\
\hline & $\begin{array}{l}\text { Serum level of } 25 \mathrm{OHD} \text { throughout } \\
\text { the study of }>50 \mathrm{ng} / \mathrm{ml}\end{array}$ & 430 & 0.29 & $0.17-0.50$ \\
\hline \multicolumn{5}{|l|}{ Cancer incidence } \\
\hline Manson et al. ${ }^{15}$ & Baseline $\mathrm{BMI}<25 \mathrm{~kg} / \mathrm{m}^{2}$ & 584 & 0.76 & $0.63-0.90$ \\
\hline
\end{tabular}

Findings are from post hoc analysis or subgroup analysis of the large clinical trials shown in TABLE 1. 25OHD, 25-hydroxyvitamin D; NM, not mentioned.

vitamin D supplementation of largely vitamin D-replete participants did not significantly reduce first or recurrent hospitalization rates for heart failure compared with no supplementation in the VITAL Heart Failure study (HR 0.93, 95\% CI 0.78-1.11; non-significant).

To date, six Mendelian randomization studies have investigated the effect of genetically altered $25 \mathrm{OHD}$ levels on cardiovascular events and related outcomes (Supplementary Box 6). These studies evaluated the effects of genetically altered 25OHD concentrations (based on two to six SNPs) in more than a million European and Chinese adults and found no significant effects on any cardiovascular event or mortality ${ }^{39-42}$. A 2020 study $^{43}$, using a substantially larger number of SNPs (242 SNPs associated with 25OHD levels adjusted for BMI, and 232 SNPs associated to 25OHD levels without adjustment for BMI), showed a non-significant odds ratio for coronary artery disease in people with genetically lowered 25OHD levels of 0.98 (95\% CI -0.06-0.02) compared with those with normal or high $25 \mathrm{OHD}$ level in a sample of 417,580 white British individuals from the UK Biobank.

Hypertension. Observational data also link hypertension with low vitamin D status but this apparent association could have been due to many other confounding factors (for example, related to lifestyle). Causal inference studies, such as RCTs and Mendelian randomization studies, should provide insights that reduce the risk of confounding. The data on blood pressure effects of vitamin D supplementation in the VITAL trial (VITAL Hypertension) are not yet available (NCT01653678; as of October 2021). The ViDA trial, however, studied extensively the effects of vitamin D supplementation in a subgroup of participants using a state of the art invasive technology (suprasystolic oscillometry) ${ }^{44}$. After a mean follow-up of 1.1 years, vitamin D supplementation generated null effects. In participants with vitamin D deficiency at baseline $(<50 \mathrm{nmol} / \mathrm{l})$, brachial systolic and diastolic blood pressure decreased by $3 \mathrm{mmHg}$ to $5 \mathrm{mmHg}$ (not statistically significant); however, aortic systolic blood pressure $(-7.5 \mathrm{mmHg}, P=0.03)$ and other parameters (augmentation index, pulse wave velocity, peak reservoir pressure and backward pressure amplitude) improved on correction of baseline vitamin D deficiency ${ }^{44}$.
The DO-HEALTH trial in European older adults did not find any effect of vitamin D supplementation on systolic or diastolic blood pressure ${ }^{18}$.

The evidence from Mendelian randomization studies on the effects of predicted serum 25OHD levels on hypertension, systolic and diastolic blood pressure is consistent across five large studies, and overall does not support any of these outcomes (Supplementary Box 6). Specifically, a study in 146,581 European individuals ${ }^{45}$, using two SNPs in the two vitamin D synthesis genes showed a marginal decrease in diastolic blood pressure of $0.29 \mathrm{mmHg}$ per $10 \%$ increase in $25 \mathrm{OHD}$ level. There was no significant effect on systolic blood pressure, and the Mendelian randomization odds ratio for hypertension was 0.92 per $10 \%$ increase in $25 \mathrm{OHD}$ level (95\% CI 0.87-0.97). A 2019 study, using six 25OHD-related $\mathrm{SNPs}^{46}$, failed to show any evidence of a causal association between 25OHD levels and systolic blood pressure, diastolic blood pressure or hypertension. Finally, using up to 252 SNPs as instruments for estimating levels of 25OHD, the most recent Mendelian randomization study in this field published in 2020 (REF ${ }^{43}$ ) showed a marginal effect of 25OHD levels on risk of hypertension (Mendelian randomization OR 0.97 per unit increase in rank-based inverse normal-transformed 25OHD level, 95\% CI 0.94-1.0) in 417,580 White British individuals from UK Biobank. After adjusting for BMI, this association became non-significant. In non-European populations, Mendelian randomization results thus far are consistent with those in Europeans. Specifically, a Mendelian randomization study ${ }^{47}$ on 2,591 Korean adults failed to show any causal effect of $25 \mathrm{OHD}$ levels on systolic blood pressure, diastolic blood pressure or risk of hypertension. A Mendelian randomization study $^{48}$ in 10,655 Chinese individuals showed equally a null effect of $25 \mathrm{OHD}$ on systolic and diastolic blood pressure.

Vitamin D and cardiovascular disease - summary. In summary, convergent evidence from Mendelian randomization studies and RCTs demonstrates that vitamin D supplementation does not decrease the risk of cardiovascular disease. The link between vitamin D status and a variety of cardiovascular events or risk factors was tested previously in mostly small-scale studies. 
The 2017-2020 megatrials (TABLE 1) and Mendelian randomization studies clearly confirm the lack of benefit of vitamin D supplementation in vitamin D-replete adults. This conclusion most likely also applies to people with vitamin $\mathrm{D}$ deficiency as based on subgroup analyses of the VITAL and ViDA trials. Unfortunately, both studies recruited very few participants with severe vitamin $\mathrm{D}$ deficiency. A dedicated detailed analysis of the ViDA trial suggested some modest benefits on central (but not peripheral) blood pressure, but the implications of this observations are limited in view of the small scale of this ViDA substudy ${ }^{44}$.

\section{Musculoskeletal effects and falls}

Vitamin D and bone health. Severe vitamin D deficiency is the leading cause of nutritional rickets ${ }^{1}$. The importance of more modest vitamin D deficiency than seen in nutritional rickets for the skeleton of adults and older adults is disputed. Supplementation with vitamin $\mathrm{D}$ only is unlikely to be able to reduce fracture risk in older adults; ${ }^{2,49}$ however, a combination of calcium and vitamin D supplementation can modestly reduce hip and non-vertebral fracture incidence in this population $^{2,50,51}$. This conclusion is in line with a 2019 overview and meta-analysis on vitamin $\mathrm{D}$ and calcium supplementation and fractures ${ }^{52}$, which concluded from observational data (39,0141 participants) that a $25 \mathrm{nmol} / 1$ increase in the serum $25 \mathrm{OHD}$ concentration reduces the risk of any fracture or hip fracture by $7 \%$ and $20 \%$, respectively (both statistically significant). A similar conclusion was reached in another meta-analysis ${ }^{53}$.

Several large RCTs have generated new results regarding the effects of vitamin D supplementation on the adult skeleton. The VITAL Bone Health study is an ancillary study of the VITAL trial, including a subcohort of 771 participants (men aged $\geq 50$ years and women aged $\geq 55$ years; not taking bone active medications) evaluated at baseline and after 2 years (89\% retention), and aims to evaluate the effects of vitamin D on bone structure and architecture. Supplemental vitamin D (compared with placebo) had no effect on 2-year changes in areal bone mineral density (BMD) at the spine, femoral neck, total hip or whole body, or on measures of bone structure. This conclusion remained valid in a subgroup analysis, including individuals with the lowest vitamin D status (as measured by total 25OHD) at baseline. New technology allows the direct measurement of free (non-protein-bound) 25OHD as an alternative strategy to define vitamin D status ${ }^{54}$. In participants of the VITAL trial with the lowest directly measured free $25 \mathrm{OHD}$ concentrations, vitamin $\mathrm{D}$ supplementation generated a slight increase in spine areal BMD $(0.75 \%$ in the vitamin $\mathrm{D}$ group versus $0 \%$ in the placebo group; $P=0.043$ ) and attenuation in loss of total hip areal BMD $(-0.42 \%$ in the vitamin $\mathrm{D}$ group versus $-0.98 \%$ in the placebo group; $P=0.044$ ), yet such results might not survive multiple testing correction ${ }^{55}$. The ViDA trial did not find an effect of monthly vitamin D supplementation on the incidence of non-vertebral fractures (RR 1.19, 95\% CI 0.94-1.50; non-significant) compared with no supplementation $^{56}$. In participants with baseline vitamin D deficiency $(<50 \mathrm{nmol} / \mathrm{l})$, the HR for non-vertebral fractures was 0.94 compared with that in vitamin D-replete participants (95\% CI 0.58-1.52). This conclusion was confirmed in the DO-HEALTH trial ${ }^{18}$.

A well-validated risk factor for fracture, such as BMD, might provide more information on the possible effects of vitamin D supplementation. In a subgroup of participants in the ViDA trial $(n=452)^{57}$, the loss of BMD during follow-up was about $0.5 \%$ lower in the vitamin $D$ group compared with the control group. This difference was statistically significant for the femoral neck and total hip but not for the lumbar spine or total body BMD. However, in the small $(n=30)$ group of participants with a baseline serum 25OHD concentration of $<30 \mathrm{nmol} / \mathrm{l}$, $\mathrm{BMD}$ of the lumbar spine increased significantly by $3.1 \%$ compared with that in controls. These data indicate that correction of severe vitamin $\mathrm{D}$ deficiency might improve bone density, but not when given to vitamin D-replete people. A smaller RCT in Scottish adults confirmed that vitamin D supplementation (daily dose of 1,000 IU) increased BMD in individuals with a baseline serum $25 \mathrm{OHD}$ concentration of $<30 \mathrm{nmol} / \mathrm{l}$ but not in people with a better vitamin $\mathrm{D}$ status at baseline $\mathrm{e}^{58}$. These results are also in line with a RCT in US adults randomized to receive placebo, $800 \mathrm{IU}$ of vitamin $\mathrm{D}$ or high-dose vita$\min \mathrm{D}$ (50,000 IU per day for 2 weeks followed by 50,000 IU per 2 weeks for 1 year), which concluded that neither low-dose nor high-dose vitamin D improved bone density in participants with a mean baseline serum $25 \mathrm{OHD}$ of $50 \mathrm{nmol} / \mathrm{l}\left(\mathrm{REF}^{59}{ }^{59}\right.$. The same conclusion was drawn from a RCT of vitamin D supplementation in Black American women, as increasing baseline serum 25OHD concentrations of $55 \mathrm{nmol} / 1$ to concentrations above $75 \mathrm{nmol} / \mathrm{l}$ by vitamin $\mathrm{D}$ supplementation did not change the rate of bone loss during 3 years of follow-up ${ }^{60}$. Similarly, Finnish children below the age of 2 years who received 1,200 IU of vitamin D per day for $\sim 2$ years did not have better bone density (measured by peripheral quantitative CT (pQCT)) compared with children receiving the standard dose of 400 IU per day ${ }^{61}$. This finding is not totally unexpected, as the baseline serum $25 \mathrm{OHD}$ concentration was higher $(80 \mathrm{nmol} / \mathrm{l})$ than expected in this study due to the introduction of vitamin D supplementation of food in Finland.

The Calgary study was designed to evaluate the effect of long-term high-dose vitamin D on bone mass and quality. A daily dose of 400 IU, 4,000 IU or 10,000 IU of vitamin $\mathrm{D}$ for 3 years in Canadian adults did not increase $\mathrm{BMD}$, but rather slightly decreased BMD, as measured by the best available methodology (high-resolution pQCT $)^{19}$. Indeed, BMD at the radius and tibia significantly decreased by $3.5 \%$ and $1.7 \%$, respectively in the $10,000 \mathrm{IU}$ per day group compared with the $400 \mathrm{IU}$ per day group, whereas the decrease at both sites was not statistically significant in the 4,000 IU per day group compared with the 400 IU per day group. This study does demonstrate that vitamin $\mathrm{D}$ supplementation in vitamin D-replete adults (baseline serum 25OHD concentration of about $75 \mathrm{nmol} / \mathrm{l})$ does not improve bone mass or quality. Moreover, very high doses might even have negative effects, as a small percentage of participants developed hypercalciuria or hypercalcaemia, which quickly resolved after adjustment of dosing. 
Of course, this finding might imply that regular follow-up is desirable when using such dosages ${ }^{19,62}$.

Many Mendelian randomization studies showed no causal effect of vitamin D status on a variety of bone traits in populations of European and non-European ancestry. An early Mendelian randomization study ${ }^{63}$ found that genetically predicted one standard deviation increase in 25OHD was not associated with increased femoral neck BMD, lumbar spine BMD or estimated BMD change. Similar results were observed in relation to total body $\mathrm{BMD}^{64}$. A more powered Mendelian randomization analysis ${ }^{65}$ (37,857 patients with fracture and 227,116 control individuals) also did not support a causal effect of 25OHD on fracture risk. However, a Mendelian randomization study in children ${ }^{66}$ showed that haplotypes associating with low 25OHD were associated with low pQCT parameters (BMD, cross-sectional area and cortical density) in 2-year-old children. Finally, evidence from Mendelian randomization studies ${ }^{67}$ refutes causal associations between predicted serum $25 \mathrm{OHD}$ concentrations and either BMD or bone metabolism markers found in 1,824 postmenopausal Chinese women (Supplementary Box 6).

Vitamin D and muscle function or falls. In mice, total deletion of VDR generates structural and functional consequences for skeletal and cardiac muscle ${ }^{9}$. Furthermore, humans with congenital CYP27B1 mutations or patients with severe combined deficiency of $25 \mathrm{OHD}$ and $1,25(\mathrm{OH})_{2} \mathrm{D}$ due to chronic renal failure develop severe muscle weakness that rapidly improves after treatment with $1,25(\mathrm{OH})_{2} \mathrm{D}^{9}$. Several meta-analyses have come to different conclusions regarding the consequences of vitamin D supplementation on muscle strength, with both positive $^{68}$ and null effects ${ }^{69}$. In addition, ample literature is available supporting a link between poor vitamin $\mathrm{D}$ status and increased risk of falls, but hesitance remains regarding causality ${ }^{70}$. High boluses of vitamin $D$, however, might transiently increase the risk of falls in older women ${ }^{71}$. High-dose continuous vitamin D supplementation to increase serum $25 \mathrm{OHD}$ concentrations to above $112 \mathrm{nmol} / \mathrm{l}$ might also induce an increased risk of falls in older men and women ${ }^{72-74}$. However, the large ViDA trial showed that monthly 100,000 IU doses of vitamin D did not reduce or increase the risk of falls. The hazard ratio for falls was 0.99 (95\% CI 0.92-1.07) in the overall cohort who were treated with vitamin D compared with those receiving placebo and 1.07 (95\% CI 0.91-1.25) in vitamin D-supplemented participants with baseline serum 25OHD concentrations below $50 \mathrm{nmol} / \mathrm{l}\left(\mathrm{REF}^{56}\right)$. The VITAL trial also looked at the effects of daily vitamin D supplementation on physical disability and falls in the SRURDY study ${ }^{75}$ and found a non-significant (OR 0.97, 95\% CI 0.91-1.25) effect of vitamin D supplementation on the risk of two falls or injurious falls requiring support from a doctor or hospital ${ }^{76}$. In further exploratory analysis, the same conclusion was reached when the baseline serum concentration of $25 \mathrm{OHD}$ was taken into account.

To our knowledge, no Mendelian randomization studies so far have examined the causal association between genetically estimated 25OHD levels and muscle traits or falls.
Vitamin D and musculoskeletal effects - summary. Of note, the 2017-2020 megatrials did not address the question of vitamin D supplementation and rickets, as there is consensus in all vitamin $\mathrm{D}$ guidelines from the past decade that serum 25OHD concentrations below $30 \mathrm{nmol} / \mathrm{l}$ are a risk factor for rickets or osteomalacia ${ }^{12}$. A daily vitamin D dose of 400 IU can prevent rickets and osteomalacia and increase serum concentrations of $25 \mathrm{OHD}$ well above $30 \mathrm{nmol} / \mathrm{l}(12 \mathrm{ng} / \mathrm{ml})^{77}$. However, $\sim 7 \%$ of the world population lives with severe vitamin D deficiency, with this percentage being much higher in the Middle East, North Africa and many countries in $\mathrm{Asia}^{78}$.

The role of vitamin $\mathrm{D}$ in the skeleton of adults and older adults is more disputed. The 2017-2020 megatrials were not designed to primarily evaluate the effect of vitamin D supplementation on fracture risk in older adults. These trials ${ }^{15,16}$ recruited mostly vitamin Dreplete adults with a fairly low risk of fracture. Even the DO-HEALTH trial in older, less vitamin D-replete, adults (compared with the other megatrials) did not find an effect on non-vertebral fractures ${ }^{18}$. However, the ViDA trial demonstrated that correction of severe vitamin D deficiency $(<30 \mathrm{nmol} / \mathrm{l})$ prevents age-related bone loss in adults. By contrast, the 2017-2020 megatrials demonstrate that vitamin $\mathrm{D}$ supplementation in vitamin $\mathrm{D}$-replete adults does not improve bone mass, density or quality ${ }^{16}$.

Taken together, the findings indicate that supplementation with vitamin D only does not have a beneficial effect on fracture risk in vitamin D-replete, mostly white adults. However, combined calcium and vitamin D supplementation in older adults, especially those with poor vitamin $\mathrm{D}$ status and poor calcium intake, might decrease the risk of hip fractures and other major fractures by about $20 \%{ }^{51}$. Therefore, most recent guidelines recommend a daily vitamin D supplement of about 800 IU of vitamin D combined with a good calcium intake (above 1,000 mg per day) in all older adults with a high risk or documented vitamin $\mathrm{D}$ deficiency. Of note, the Calgary study demonstrated that high daily doses of vitamin D (4,000 and especially 10,000 IU per day) might decrease BMD and bone quality ${ }^{19,20}$. Therefore, the optimal dose in vitamin $\mathrm{D}$-deficient older adults should be at least $800 \mathrm{IU}$ per day but not more than 4,000 IU per day.

Meta-analyses of older studies suggested a modest decrease in the risk of falls in older, mostly vitamin D-deficient, adults ${ }^{79}$. However, the ViDA trial did not confirm this finding as vitamin D supplementation did not change the risk of falls. The New Zealand population was younger and had a better vitamin $\mathrm{D}$ status than the participants in the older studies. There might also be a U-shaped relationship as very high vitamin status, especially due to high bolus doses, might increase the risk of falls ${ }^{72-74}$.

\section{Lung function and respiratory effects}

Vitamin D and respiratory infections or lung function. The lung is increasingly recognized as an important target tissue for vitamin D. Observational data link poor vitamin $\mathrm{D}$ status with several inflammatory lung diseases or impaired lung function ${ }^{80-82}$. The most recent analysis published in 2019 (REF. ${ }^{83}$ ) evaluated 10,933 participants 
in 25 RCTs and found a significant overall reduction in acute respiratory infections following vitamin $\mathrm{D}$ supplementation (OR 0.88, 95\% CI 0.81-0.96) compared with no supplementation. The number needed to treat for benefit was 33. Subgroup analysis revealed that the greatest benefits were found in people with severe vitamin D deficiency $(<25 \mathrm{nmol} / \mathrm{l})$ at baseline (OR 0.58 , $95 \%$ CI $0.40-0.82$ ). Subgroup analysis revealed that intermittent (monthly or less frequent) doses of vitamin $\mathrm{D}$ did not generate protection, whereas daily or weekly vitamin D supplementation was more effective for preventing acute respiratory infections (OR $0.81,95 \% \mathrm{CI}$ $0.072-0.91)$. In the ViDA trial, however, no effects of vitamin D supplementation were found on acute respiratory infections in older adults ${ }^{84}$. This finding is not a total surprise as the lack of effects might be due to the intermittent dosing and/or adequate vitamin $\mathrm{D}$ status at baseline, and therefore might not contradict the findings of the 2019 meta-analysis ${ }^{83}$. In addition, the European DO-HEALTH trial did not show an effect on infections in general nor on upper respiratory infections ${ }^{18}$.

Several small-scale studies (eight RCTs) did not find an improvement in lung function (as measured in terms of forced expiratory volume in $1 \mathrm{~s}$ (FEV1)) in patients with chronic obstructive pulmonary disease (COPD) who were randomized to receive vitamin $\mathrm{D}$ supplementation ${ }^{85}$. A substudy of the ViDA trial, however, evaluated the effects of monthly vitamin D supplementation ${ }^{86}$ in 442 adults treated for 1.1 years. Overall, in the ITT analysis, no significant effects were observed on FEV1. However, subgroup analysis revealed some beneficial effects, especially in subjects with existing lung problems such as asthma, COPD or a history of smoking (TABLE 2). To date, no Mendelian randomization studies have been performed that examined 25OHD levels, COPD and lung function.

Vitamin D and COVID-19. In view of the enormous health implications of the coronavirus disease 19 (COVID-19) pandemic caused by the worldwide spread of severe acute respiratory syndrome coronavirus 2 , a possible link with poor vitamin D status and the risk or severity of COVID-19 has received great attention. Seven studies so far compared serum $25 \mathrm{OHD}$ concentrations in patients with COVID-19 compared with individuals without COVID-19 $\left(\mathrm{REF}^{87}\right)$ and found a lower level (mean difference of about $12 \mathrm{nmol} / \mathrm{l}$ ) in patients with COVID-19; however, in many studies the sampling did not take place at the same time in both groups. In addition, these studies were unable to control for confounding factors, a major problem due to the large number of similarities in the risk factors for vitamin D deficiency and COVID-19. About 31 studies looked at a possible link between vitamin D status and severity of the outcome of COVID-19. Lower serum concentrations of $25 \mathrm{OHD}$ were associated with greater mortality, greater need for intensive care treatment or increased severity of illness in general compared with better vitamin D status. However, this finding was based on observational studies. One placebo-controlled intervention study using a bolus dose of vitamin D (200,000 IU) did not reveal a beneficial effect in patients hospitalized with COVID-19 with a mean baseline
$25 \mathrm{OHD}$ concentration of $50 \mathrm{nmol} / \mathrm{l}\left(\mathrm{REF}^{88}\right)$. However, one pilot study (which was not placebo-controlled) showed a marked reduction in the need for intensive care treatment in patients hospitalized for COVID-19 and treated with a high dose of $25 \mathrm{OHD}$ (calcifediol) at the time of admission $^{89}$. Therefore, the link between vitamin $\mathrm{D}$ status and COVID-19 is unsettled so far, but many trials are ongoing that might clarify this question.

In 2021, a Mendelian randomization study assessed the causal role of serum 25OHD levels on COVID-19 susceptibility and disease severity ${ }^{90}$. Using data from 11,181 patients with COVID-19 and 116,456 control individuals from the Host Genetics Initiative, and six vitamin D SNPs that explain $2.5 \%$ of the variance in serum $25 \mathrm{OHD}$ levels, this study did not show any association between genetically decreased 25OHD and COVID-19 susceptibility or severity. These results were confirmed in a separate Mendelian randomization study using $8125 \mathrm{OHD}$ SNPs that explain $4.3 \%$ of the variance in serum $25 \mathrm{OHD}$ levels, which also showed no effect of genetically determined 25OHD levels on risk of COVID-19-related hospitalization ${ }^{91}$.

Vitamin D and asthma. Research investigating the potential effects of vitamin D status on asthma has largely focused on a possible link between prenatal or maternal vitamin $\mathrm{D}$ status and wheezing or asthma in the offspring. A meta-analysis of four prospective studies and three RCTs concluded that vitamin D intake ( 800 IU per day) by women during pregnancy is inversely related to wheezing or asthma in their offspring during up to 3 years of follow-up ${ }^{92}$. However, a longer follow-up did not confirm this conclusion: vitamin D supplementation during the prenatal period alone did not influence the 6-year incidence of asthma and recurrent wheeze among children who were at risk of asthma ${ }^{93}$. Two Mendelian randomization studies have investigated the causal association between vitamin $\mathrm{D}$ and asthma. A large study ( $\mathrm{n}>160,000$ children and adults) ${ }^{94}$ found odds ratios of 1.03 (95\% CI 0.90-1.19) for asthma and 0.95 (95\% CI 0.69-1.31) for childhood-onset asthma per standard deviation of log-transformed decrease in serum 25OHD (Supplementary Box 6). These findings suggest that vitamin D levels probably do not have clinically relevant effects on the risk of asthma.

Vitamin D and respiratory effects - summary. The vitamin D endocrine system influences all cells and most cytokines of the immune system ${ }^{9}$. The innate immune system is stimulated by $1,25(\mathrm{OH})_{2} \mathrm{D}$ and this is in line with a decreased risk of upper respiratory infections with vitamin D supplementation in individuals with vitamin $\mathrm{D}$ deficiency ${ }^{83}$. Meta-analysis of intervention studies suggested a benefit of vitamin D supplementation of participants with severe vitamin D deficiency and COPD, asthma, or similar lung diseases, and on reducing the risk of acute upper respiratory infections in severely deficient individuals ${ }^{83}$. However, where tested, these findings have not been supported by Mendelian randomization studies $^{90}$. According to the results of the LUNG-ViDA trial, vitamin D supplementation might modestly improve expiratory lung function ${ }^{85}$. If confirmed, such 


\begin{tabular}{|c|c|c|c|c|c|c|}
\hline $\begin{array}{l}\text { Study and } \\
\text { ref. }\end{array}$ & Genes $^{a}$ & $\begin{array}{l}\text { Number of } \\
\text { controls; } \\
\text { number of cases }\end{array}$ & Outcome & $\begin{array}{l}\text { Estimate } \\
\text { of effect } \\
\text { (OR and } \\
95 \% \mathrm{Cl})\end{array}$ & $P$ value & $\begin{array}{l}\text { Unit of } \\
\text { estimated effect }\end{array}$ \\
\hline Mokry et al. ${ }^{96}$ & $\begin{array}{l}\text { DHCR7, } \\
\text { CYP2R1, } \\
\text { GC and } \\
\text { CYP24A1 }\end{array}$ & 38,$589 ; 14,498$ & Multiple sclerosis & $\begin{array}{l}2.02 \\
(1.65-2.46)\end{array}$ & $7.7 \times 10^{-12}$ & $\begin{array}{l}\text { One standard } \\
\text { deviation } \\
\text { decrease in } \\
\text { log-transformed } \\
25 O H D \text { level }\end{array}$ \\
\hline Rhead et al..$^{99}$ & $\begin{array}{l}\text { DHCR7, } \\
\text { CYP2R1 } \\
\text { and GC }\end{array}$ & 22,$168 ; 7,391$ & Multiple sclerosis & $\begin{array}{l}0.85 \\
(0.76-0.94)\end{array}$ & 0.003 & $\begin{array}{l}\text { Unit change } \\
\text { in polygenic } \\
\text { risk score }\end{array}$ \\
\hline Jacobs et al. ${ }^{97}$ & $\begin{array}{l}\text { CYP2R1, } \\
\text { DHCR7, } \\
\text { CYP24A1, } \\
\text { SEC23A and } \\
\text { AMDHD1 }\end{array}$ & 41,$505 ; 14,802$ & Multiple sclerosis & $\begin{array}{l}0.57 \\
(0.41-0.81)\end{array}$ & 0.001 & $\begin{array}{l}\text { One unit increase } \\
\text { in natural } \\
\text { log-transformed } \\
\text { vitamin D level }\end{array}$ \\
\hline $\begin{array}{l}\text { Gianfrancesco } \\
\text { et al. }{ }^{.8}\end{array}$ & $\begin{array}{l}\text { GC, CYP2R1 } \\
\text { and DHCR7 }\end{array}$ & 1,$715 ; 34$ & $\begin{array}{l}\text { Paediatric-onset } \\
\text { multiple sclerosis }\end{array}$ & $\begin{array}{l}0.72 \\
(0.55-0.94)\end{array}$ & 0.02 & NA \\
\hline
\end{tabular}

25OHD, 25-hydroxyvitamin D; NA, not applicable. ${ }^{a} G e n e s$ in which variants can be used to infer serum 25OHD concentration.

data would imply that the lung is a clinically relevant target issue for vitamin $\mathrm{D}$. Of note, currently there are insufficient RCTs to evaluate the potential benefit of vitamin $\mathrm{D}$ or calcifediol supplementation on the risk or severity of COVID-19.

\section{Autoimmune diseases}

Observational studies have, in line with preclinical data, made a link between poor vitamin D status and increased risk of infection or risk of autoimmune diseases (such as multiple sclerosis (MS), inflammatory bowel diseases or type 1 diabetes mellitus ${ }^{95}$. RCTs in humans dealing with infections have mainly focused on upper respiratory infections and an overview is presented in the previous section. Unfortunately, no major RCTs have addressed the possible primary or secondary prevention of the major human autoimmune diseases. So far, the 2017-2020 megatrials (TABLE 1) have not shown results related to autoimmune diseases.

Currently, strong evidence exists that supports a causal association between genetically low serum 25OHD levels and increased risk of $\mathrm{MS}^{96-99}$. The most recent Mendelian randomization study from 2020 evaluated data from The International Multiple Sclerosis Genetics Consortium discovery phase GWAS (14,802 MS and 26,703 controls from the USA, Europe, Australia and some Asian countries $)^{97}$ using six SNPs associated with serum levels of $25 \mathrm{OHD}$ and found that each genetically determined unit increase in log-transformed $25(\mathrm{OH}) \mathrm{D}_{3}$ was associated with an odds ratio for MS of 0.57 (95\% CI 0.41-0.81; $P=0.001$ ) (TABLE 4). This effect applies to adult-onset and childhood-onset MS.

Earlier Mendelian randomization evidence ${ }^{100} \mathrm{did}$ not support causality of predicted serum 25OHD levels in systemic lupus erythematosus or rheumatoid arthritis. Consistent null effects on rheumatoid arthritis were found in a 2020 Mendelian randomization study in participants from the UK Biobank, using 220 vitamin $\mathrm{D}$-associated SNPs as instruments ${ }^{43}$. Null effects of predicted serum 25OHD levels were also shown in Mendelian randomization studies on Crohn's disease (odds ratio for $10 \mathrm{nmol} / \mathrm{l}$ higher $25 \mathrm{OHD}$ of $1.04,95 \%$ CI $0.93-1.16)$ and ulcerative colitis (OR 1.13, 95\% CI 1.06-1.21) ${ }^{101}$. Similarly, no effect on ulcerative colitis was found in participants from the UK Biobank ${ }^{43}$. The UK Biobank study also did not support a causal role of vitamin $\mathrm{D}$ on allergic rhinitis. Finally, Mendelian randomization ${ }^{94}$ does not support causal effects of 25OHD on atopic dermatitis. A 2021 Mendelian randomization study on type 1 diabetes mellitus did not support causal effects of genetically lowered $25 \mathrm{OHD}$ levels on the risk of this disease ${ }^{102}$.

In summary, the adaptive immune system is downregulated by $1,25(\mathrm{OH})_{2} \mathrm{D}$ and therefore vitamin $\mathrm{D}$ deficiency might predispose to autoimmune diseases ${ }^{9}$. Observational studies have suggested this effect might apply to humans, but too few intervention studies have been conducted to evaluate this statement. Four independent Mendelian randomization studies agree, however, that individuals with genetically driven lower serum 25OHD concentrations have an increased risk of developing MS, either during adolescence or adulthood (TABLE 4).

\section{Pregnancy}

Intervention studies as summarized in a Cochrane review from 2016 (REF. ${ }^{103}$ ) dealing with 22 RCTs including 3,725 pregnant women, concluded that vitamin D supplementation significantly reduced the risk of pre-eclampsia (RR 0.48), gestational diabetes mellitus (RR 0.51) and low birthweight (<2,500 g; RR 0.55) compared with no supplementation. An update of these data $^{104}$ largely confirmed these observations. However, a large RCT in pregnant Bangladeshi women with severe vitamin D deficiency (baseline mean serum 25OHD about $25 \mathrm{nmol} / \mathrm{l}$ ) supplemented from week 17-24 onwards with placebo or vitamin D (three groups receiving 4,200,16,800 or 28,000 IU per week) until birth did not find a beneficial effect on fetal or neonatal parameters of length, weight or head circumference, either at birth or at one year of age $(n=1,164 \text { infants })^{105}$. 
To date only one Mendelian randomization study ${ }^{106}$ has examined the causal effect of predicted serum 25OHD levels on gestational hypertension and pre-eclampsia. Overall, the evidence was weak supporting a causal effect of vitamin D status on gestational hypertension (OR $0.90,95 \%$ CI $0.78-1.03$ ) or pre-eclampsia (OR 0.98 , 95\% CI: $0.89-1.07$ ) per $10 \%$ decrease in serum 25OHD (Supplementary Box 5).

In summary, pregnant women more frequently have a poor vitamin $\mathrm{D}$ status than non-pregnant women of the same age but the absolute and relative values vary from country to country. Several meta-analyses have suggested that vitamin D supplementation might modestly decrease maternal morbidity and improve the health of their offspring ${ }^{103,104}$. However, a 2018 large RCT in Bangladeshi women with severe vitamin D deficiency did not confirm this observation ${ }^{105}$. Therefore, the effects of poor vitamin $\mathrm{D}$ status during pregnancy on pregnancy outcomes for mother and infant remains unsettled.

\section{Patients in intensive care}

Patients with severe acute illness requiring intensive care frequently have low serum concentrations of $25 \mathrm{OHD}$ and this poor vitamin D status is linked with increased morbidity and mortality ${ }^{107,108}$. Two major RCTs so far in patients in intensive care units (ICU) have generated conflicting results. In the VITdAL-ICU trial, patients in the ICU were randomized to either placebo $(n=243)$ or high-dose oral vitamin D $(n=249)$ (starting dose 540,000 IU followed by monthly maintenance doses of 90,000 IU for 5 months). Mean baseline serum 25OHD concentrations were low $(33 \mathrm{nmol} / \mathrm{l})$ and increased to $\sim 82 \mathrm{nmol} / \mathrm{l}$ at day 3 . Length of stay in the ICU or hospital, mortality in the ICU, in-hospital mortality and mortality at 6 months did not improve with the intervention. In a predefined subgroup with severe vitamin $\mathrm{D}$ deficiency who received the intervention $(<30 \mathrm{nmol} / \mathrm{l})$, hospital mortality (HR 0.56, 95\% CI, 0.35-0.90) and 6-month mortality (HR $0.60,95 \%$ CI, $0.39-0.93$ ) were significantly decreased compared with patients with severe vitamin D deficiency who received placebo ${ }^{107}$. In the much larger Amrein ICU trial ${ }^{108}, 1,059$ patients in the ICU with vitamin D deficiency $(<50 \mathrm{nmol} / \mathrm{l})$ received either placebo or a single oral high dose of vitamin D (540,000 IU). This dose increased mean serum $25 \mathrm{OHD}$ concentration at day 3 to a mean concentration of $117 \pm 58 \mathrm{nmol} / \mathrm{l}$ in comparison with the control group (mean concentration $28 \pm 14 \mathrm{nmol} / \mathrm{ml}$ ). The primary end point (90-day mortality) and other non-fatal outcomes were similar in the two groups. Although all patients in both studies were admitted to ICUs, the US patients in the VITdAL-ICU trial were probably less sick than those in the Amrein trial ${ }^{107}$ as indicated by the percentage of patients requiring mechanical ventilation (32\% in the US trial).

\section{Effects of vitamin D supplementation on safety outcomes}

In all vitamin D supplementation RCTs, some safety end points have been reported in addition to mortality (see next section). No effects were found on serum calcium or calciuria unless very high doses were used, such as 4,000-10,000 IU per day in the Calgary study.
Even in these circumstances, hypercalcaemia was infrequent and occurred transiently after changes in treatment modality ${ }^{1962}$. A modestly increased risk of kidney stones was observed in the WHI trial ${ }^{109}$, but this effect was not seen in the more recent 2017-2020 megatrials (that is, ViDA, VITAL and D2d; TABLE 1). Furthermore, no changes in kidney function were found in these large trials. Skeletal consequences were either null effects, slight (beneficial) increases in BMD in subgroups with poor vitamin $\mathrm{D}$ status at baseline, or a modest but significant decrease in BMD during high-dose (10,000 IU per day) therapy in the Calgary study ${ }^{19}$. An increased risk of fractures in patients receiving high intermittent bolus doses has been reported ${ }^{71,110}$. Similarly, an increased risk of falls has been reported when either high intermittent doses ${ }^{71}$ or high continuous doses were used $\mathrm{d}^{72,73}$. Importantly, the 2017-2020 megatrials (that is, ViDA, VITAL and D2d), with detailed evaluation of about 30,000 participants for 2-5 years, did not discover notable adverse effects. These findings indicate that a daily dose (or dose equivalent) of 2,000-4,000 IU can be considered as safe in an adult (even vitamin D-replete) population. High-dose vitamin $\mathrm{D}$ also did not modify arterial calcifications during a 3-year follow up in the Calgary study ${ }^{111}$.

\section{Effects of vitamin D supplementation on mortality}

Observational data have repeatedly linked poor vitamin D status with increased mortality. This effect was extensively documented in several NHANES studies based on representative samples of the US population and confirmed after validation of serum 25OHD concentrations according to standards generated by the US National Institute of Standards and Technology ${ }^{112}$. To decrease the possible effect of reverse causation, people who died within the first 3 years after 25OHD measurements were excluded from the analysis; however, the same association between poor vitamin $\mathrm{D}$ status and increased mortality remained ${ }^{112}$. Using a combination of several European prospective studies, mortality was also higher in the population with the poorest vitamin D status compared with the vitamin D-replete population ${ }^{113}$. A 2019 large long-term ( $>10$ years) Finnish study concluded that people with the highest tertile of $25 \mathrm{OHD}$ concentrations $(>50 \mathrm{nmol} / \mathrm{l})$ had a mortality odds ratio of 0.77 (95\% CI 0.71-0.84) compared with people with the lowest tertile of $25 \mathrm{OHD}$ concentrations, even in a multivariate model with correction of multiple co-variables ${ }^{114}$.

As nearly all long-term vitamin D supplementation trials include data on mortality, several meta-analyses have shown the effects of vitamin D supplementation on mortality. Extensive meta-analyses published in 2014 showed a modest decrease in overall mortality in participants randomized to vitamin D supplementation; based on 22 RCTs, the risk of death decreased by $11 \%{ }^{36}$. A 2014 Cochrane analysis ${ }^{29}$ evaluated 56 RCTs including 95,286 participants (mostly healthy women older than 70 years) with a mean follow-up of 4.4 years. Vitamin D supplementation significantly reduced all-cause mortality (RR 0.94, 95\% CI 0.91-0.98; $P=0.002$ ) compared with no supplementation. This finding implies that vitamin $\mathrm{D}$ supplementation of 150 women for 5 years prevented 
one additional death. Vitamin D supplementation also decreased cancer mortality (RR 0.88, 95\% CI 0.78-0.98; $P=0.02)$ compared with no supplementation ${ }^{29}$.

In the 2017-2020 megatrials (that is, VITAL, ViDA and D2d), overall mortality was much lower than shown in the previous meta-analyses ${ }^{29,36}$ and did not show an effect of vitamin D supplementation on overall mortality ${ }^{15}$. A new meta-analysis of 52 RCTs including a total of 75,454 participants concluded that vitamin D (either vitamin $\mathrm{D}_{3}$ or $\mathrm{D}_{2}$ ) supplementation did not change mortality (RR 0.98, 95\% CI 0.95-1.02) compared with no supplementation ${ }^{115}$. A subanalysis, however, found that vitamin $\mathrm{D}_{3}$ (instead of $\mathrm{D}_{2}$ ) supplementation trials tended to reduce mortality (RR $0.95,95 \%$ CI $1.90-1.00 ; P=0.06$ ), whereas this was not the case for vitamin $\mathrm{D}_{2}$ supplementation trials. These new findings conflict with the 2014 reports $^{112}$. The difference could be partly because the 2019 meta-analysis did not include ten RCTs including 50,000 participants using a combination of vitamin $\mathrm{D}$ and calcium supplementation. However, the 2019 meta-analysis did include two megatrials (VITAL and ViDA) that evaluated the effects of vitamin $\mathrm{D}$ supplementation in a younger population of mostly vitamin D-replete participants ${ }^{115}$.

In a large-scale population Mendelian randomization study $(10,349 \text { deaths in } 95,766 \text { total participants })^{116}$, the odds ratios for a genetically determined lower 25OHD concentration was 1.30 (95\% CI 1.05-1.61) for all-cause mortality, 0.77 (95\% CI 0.55-1.08) for cardiovascular mortality, 1.43 (95\% CI 1.02-1.99) for cancer mortality and 1.44 (95\% CI 1.01-2.04) for other types of mortality. Similar point estimates and effect sizes, whose $95 \%$ confidence intervals included the null, were found for all-cause mortality in two follow-up Mendelian randomization studies ${ }^{46,117}$. Nevertheless, both studies may have been underpowered to detect existing causal associations. Finally, evidence from Mendelian randomization ${ }^{118}$ did not support an association between 25OHD concentrations and cancer mortality in a sample of 6,998 deaths from cancer. These data provide some evidence that genetically lowered vitamin D levels might increase overall mortality risks, but the results have not been consistent across studies, or across causes of mortality.

If vitamin D supplementation exerts beneficial effects on extra-skeletal health outcomes and major diseases, then it is likely to have some effects on mortality, especially in older adults with poor vitamin D status. Large meta-analyses dealing mostly with women older than 70 years $^{29,36}$ showed a $6-11 \%$ reduction in mortality; however, adding the newest 2017-2020 megatrials eliminated this effect, possible because these new trials recruited a younger population.

\section{Discordance between studies}

Preclinical data are mostly in line with the very large number of observational studies linking very poor vitamin D status with skeletal and extra-skeletal health effects (FIG. 1). However, Mendelian randomization studies and the majority of RCTs do not confirm the causality of these associations. Several possible reasons exist for this discrepancy. Most importantly, serum 25OHD levels are a highly confounded variable. Specifically, serum
25OHD levels are affected by a host of health behaviours, the presence of obesity, socioeconomic status and education levels. Although most observational studies have attempted to control for such confounding through multivariable adjustment, such approaches depend upon the degree of accuracy of measurement of the confounders, knowledge that such confounding takes place, and most often that the nature of the confounding relationship (linear versus nonlinear) is known. Furthermore, statistical adjustment for confounding variables can only be accomplished if the confounding variables are known.

The concordance between 25OHD Mendelian randomization studies and RCTs is striking and suggests that Mendelian randomization might be a more relevant way to begin to understand the effect of $25 \mathrm{OHD}$ levels on risk of disease than observational studies. Perhaps the vitamin D endocrine system only has a role in these extra-skeletal effects in people with prolonged and very severe vitamin D deficiency. Studies in countries or population groups with severe vitamin D deficiency who need improved vitamin D status anyway might be the ideal approach to better understand the effect of vitamin D supplementation in individuals with severe vitamin D deficiency. Most RCTs and Mendelian randomization studies have been undertaken in individuals from the general population in which the rates of severe vitamin $\mathrm{D}$ deficiency are low.

Of note, the available Mendelian randomization studies were not able to predict large variations in serum $25 \mathrm{OHD}$ concentrations (usually only about $5 \%$ difference or less). However, this low degree of variance would affect the statistical power of a study but not introduce bias. New techniques will soon enable us to use a much larger number of SNPs than used in current studies (usually based on less than six SNPs), thereby allowing much larger variations in serum $25 \mathrm{OHD}$ concentrations to be predicted. Most RCTs did not last longer than 3-5 years. In such short-term scenarios, answering the question of causality is extremely difficult. This fact implies that only very long-term improvements in vitamin D status might generate beneficial effects. However, Mendelian randomization studies provide estimates of the effect of a lifetime of genetically lowered vitamin D levels and such Mendelian randomization studies have generally produced null findings.

Reverse causality remains a valid rationale to explain the discordance between observational and intervention studies. The most plausible hypothesis states that individuals with any health problems are less likely to regularly engage in outdoor activity and less exposure to sunlight results in lower vitamin D status. Another mechanism of reverse causality might be that the activity of hepatic 25 -hydroxylase is decreased in many major diseases and this decrease could cause low serum 25OHD concentrations. Indeed, data in mice demonstrate that diet-induced obesity, type 1 diabetes mellitus or T2DM, fasting and exposure to glucocorticoids substantially decrease the gene and protein expression of CYP2R1, thereby decreasing the overall 25 -hydroxylase activity ${ }^{119-121}$. This finding implies that decreased $25 \mathrm{OHD}$ concentrations are the consequence of disease, rather than involved in the origin of these metabolic diseases. Of course, these 
data from mice need confirmation in humans. Finally, many diseases other than those described in this Review (including brain-related diseases) are linked with poor vitamin D status; however, causality is doubtful without adequate Mendelian randomization studies or RCTs.

\section{Future Mendelian randomization studies}

Improved understanding of the genetic determinants of $25 \mathrm{OHD}$ has helped re-assess the role of vitamin $\mathrm{D}$ in the aetiology of complex diseases through Mendelian randomization. Taken together, the evidence from over 60 Mendelian randomization studies published to date assessing the role vitamin D does not support a causal role for the large majority of studied outcomes. Despite this null data, in the few cases where the evidence from Mendelian randomization supported a causal role of vitamin D status, such as in the example of MS, these results had important clinical implications. For instance, clinical care guidelines for the use of vitamin D in preventing MS in those at risk were published by the MS Society of Canada ${ }^{122}$.

The earlier Mendelian randomization studies used, as instruments for 25OHD levels, SNPs within the four genes related to 25OHD synthesis and metabolism (DHCR7, CYP2R1, GC and CYP24A1), which together explained $2.4 \%$ of the variance in $25 \mathrm{OHD}$ levels ${ }^{123}$. Later Mendelian randomization studies combined the aforementioned four SNPs with two SNPs in SEC23A and $A M D H D 1$ (both genes without clear role in the vitamin D metabolic pathway), and thereby explained $\sim 5.3 \%$ of the variance in $25 \mathrm{OHD}$ levels. The identification of over 150 25OHD-associated genetic variants in 2020, which explain a considerable portion of the variance in $25 \mathrm{OHD}$ levels $(\sim 10.5 \%)^{43}$, has enabled a deeper understanding of the genetic determinants contributing to variation in circulating 25OHD levels. These newly identified SNPs will probably enable improved instrumentation of vitamin D in Mendelian randomization studies. Moreover, with the emergence of large-scale GWAS in densely phenotyped biobanks, we anticipate that more powerful vitamin D Mendelian randomization studies will be published that utilize the optimized set of genetic instruments. Such new studies should revisit previously studied diseases and investigate new disease outcomes, to further aid causal effect estimation.

\section{Conclusions}

In conclusion, the data generated by the 2017-2020 megatrials of vitamin D supplementation in largely vitamin D-replete adults (TABLE 1) demonstrate that increasing the serum 25OHD concentration into the high normal range (based on the IOM and most recent guidelines published over the past decade ${ }^{12}$, in the range of 50-125 $\mathrm{nmol} / \mathrm{l}$ or $20-50 \mathrm{ng} / \mathrm{ml}$ ) does not generate benefits for global health or major diseases or medical events such as cancer, cardiovascular events, T2DM, falls or fractures. Therefore, no reason exists at present to recommend vitamin $\mathrm{D}$ supplementation of already vitamin $\mathrm{D}$-replete individuals. These data do not contradict the causal link between severe vitamin D deficiency and rickets, or the need to correct severe deficiency at any age. Similarly, the 2017-2020 trials do not contradict the probable beneficial effects of combined supplementation of calcium and vitamin $\mathrm{D}$ in older adults with poor vitamin $\mathrm{D}$ and calcium status on their risks of fracture or falls.

A few hints have emerged that vitamin D supplementation might have some extra-skeletal benefits, especially in people with severe vitamin D deficiency (such as reduced progression to T2DM, decreased numbers of infections, increased lung function and decreased cancer or overall mortality) (TABLES 2,3). These suggestions are largely based on subgroup or post hoc analyses and thus should not result in the systematic recommendation of vitamin $\mathrm{D}$ supplements in such populations but might guide the correct design of future studies.

Arguments have been put forward that daily doses of $\geq 4,000$ IU of vitamin D convey some risks other than simple hypercalcaemia or hypercalciuria. Such doses, or the equivalent of serum $25 \mathrm{OHD}$ concentrations well above $112 \mathrm{nmol} / \mathrm{l}$ or $45 \mathrm{ng} / \mathrm{ml}$ bring no benefits, but might be harmful in some people (for example, in causing loss of BMD or increasing the risk of falls). The same is true for intermittent high-dose boluses of vitamin D. Unfortunately, about $3 \%$ of the US population as screened by NHANES use such high dose vitamin D supplements.

Over the past few decades, vitamin $\mathrm{D}$ has been a hot topic for scientists and lay people alike, who frequently suggest that vitamin D supplementation might generate a wide variety of health benefits. The data discussed in the present Review might well dampen such enthusiasm. However, a large number of intervention studies (and most probably Mendelian randomization studies) are still ongoing, and these might help provide a better understanding of who would benefit from vitamin D supplementation.

In conclusion, it seems that far too many people with severe vitamin D deficiency ( $7 \%$ of the world population) do not take or even have access to normal doses of vitamin D. About a third of the world population lives with suboptimal (below $20 \mathrm{ng} / \mathrm{ml}$ ) serum 25OHD concentrations $^{78}$. However, many vitamin D-replete people take vitamin $\mathrm{D}$ supplements without clear benefits. In addition, a small percentage of the population takes higher doses than the upper limit of safe intake. Therefore, we recommend that vitamin D be used wisely and "giveth to those who needeth"

Published online 23 November 2021
1. Munns, C. F et al. Global consensus recommendations on prevention and management of nutritional rickets. J. Clin. Endocrinol. Metab. 101, 394-415 (2016).

2. Bolland, M. J., Grey, A. \& Avenell, A. Effects of vitamin D supplementation on musculoskeletal health a systematic review, meta-analysis, and trial sequential analysis. Lancet Diabetes Endocrinol. 6, 847-858 (2018).
3. Luxwolda, M. F., Kuipers, R. S., Kema, I. P. Dijck-Brouwer, D. A. \& Muskiet, F. A. Traditionally living populations in East Africa have a mean serum 25-hydroxyvitamin D concentration of $115 \mathrm{nmol} / \mathrm{l} . \mathrm{Br}$. J. Nutr. 108, 1557-1561 (2012).

4. Luxwolda, M. F. et al. Vitamin D status indicators in indigenous populations in East Africa. Eur. J. Nutr. 52 1115-1125 (2013)
5. Holick, M. F. \& Grant, W. B. Vitamin D status and ill health. Lancet Diabetes Endocrinol. 2, 273-274 (2014).

6. Bouillon, R., Lips, P. \& Bilezikian, J. P. Vitamin D supplementation and musculoskeletal health. Lancet Diabetes Endocrinol 7, 85-86 (2019).

7. Lips, P., Bilezikian, J. P. \& Bouillon, R. Vitamin D: giveth to those who needeth. JBMR 4, e 10232 (2020). 
8. Ebeling, P. R. et al. Management of endocrine disease: therapeutics of vitamin D. Eur. J. Endocrinol. 179 R239-R259 (2018)

9. Bouillon, R. et al. Skeletal and extraskeletal actions of vitamin D: current evidence and outstanding questions. Endocr. Rev. 40, 1109-1151 (2019).

10. Institute of Medicine. Dietary Reference Intakes for Calcium and Vitamin D (National Academies Press, 2011)

11. Holick, M. F. et al. Evaluation, treatment, and prevention of vitamin D deficiency: an Endocrine Society clinical practice guideline. J. Clin. Endocrinol. Metab. 96, 1911-1930 (2011)

12. Bouillon, R. Comparative analysis of nutritional guidelines for vitamin D. Nat. Rev. Endocrinol. 13 466-479 (2017)

13. Scientific Advisory Committee on Nutrition (SACN). Vitamin D and health. GOV.UK https://www.gov.uk/ government/publications/sacn-vitamin-d-and-healthreport (2016)

14. Lips, P. et al. Current vitamin D status in European and Middle East countries and strategies to prevent vitamin $D$ deficiency; a position statement of the European Calcified Tissue Society. Eur. J. Endocrinol. 180, 23-54 (2019).

15. Manson, J. E. et al. Vitamin D supplements and prevention of cancer and cardiovascular disease. N. Engl. J. Med. 380, 33-44 (2019).

16. Scragg, R. et al. Monthly high-dose vitamin D supplementation and cancer risk: a post hoc analysis of the vitamin $\mathrm{D}$ assessment randomized clinical trial. JAMA Oncol. 4, e182178 (2018).

17. Pittas, A. G. et al. Vitamin D supplementation and prevention of type 2 diabetes. N. Engl. J. Med. 381 , 520-530 (2019)

18. Bischoff-Ferrari, H. A. et al. Effect of vitamin D supplementation, omega-3 fatty acid supplementation, or a strength-training exercise program on clinical outcomes in older adults: the DO-HEALTH Randomized Clinical Trial. JAMA 324, 1855-1868 (2020).

19. Burt, L. A. et al. Effect of high-dose vitamin D supplementation on volumetric bone density and bone strength: a randomized clinical trial. JAMA 322 , 736-745 (2019).

20. Billington, E. O. et al. Safety of high-dose vitamin D supplementation: secondary analysis of a randomized controlled trial. J. Clin. Endocrinol. Metab. 105 1261-1273 (2020)

21. Dawson-Hughes, B. et al. Intratrial exposure to vitamin $\mathrm{D}$ and new-onset diabetes among adults with prediabetes: a secondary analysis from the Vitamin $D$ and Type 2 Diabetes (D2d) Study. Diabetes Care 43 2916-2922 (2020).

22. Pittas, A G Jorde, R., Kawahara, T. $\varnothing$ Dawson-Hughes, B. Vitamin D supplementation for prevention of type 2 diabetes mellitus: to $\mathrm{D}$ or not to D? J. Clin. Endocrinol. Metab. 105, 3721-3733 (2020).

23. Zhang, Y. et al. Effects of Vitamin D supplementation on prevention of type 2 diabetes in patients with prediabetes: a systematic review and meta-analysis. Diabetes Care 43, 1650-1658 (2020).

24. Barbarawi, M. et al. Effect of vitamin D supplementation on the incidence of diabetes mellitus. J. Clin. Endocrinol. Metab. 105, 2857-2868 (2020).

25. Lu, L. et al. Association of vitamin D with risk of type 2 diabetes: a Mendelian randomisation study in European and Chinese adults. PLoS Med. 15 e1002566 (2018).

26. Diabetes Prevention Program Research Group. Long-term effects of lifestyle intervention or metformin on diabetes development and microvascular complications over 15-year follow-up: the Diabetes Prevention Program Outcomes Study. Lancet Diabetes Endocrinol. 3, 866-875 (2015).

27. Feldman, D., Krishnan, A. V., Swami, S., Giovannucci, E. \& Feldman, B. J. The role of vitamin D in reducing cancer risk and progression. Nat. Rev. Cancer 14 342-357 (2014)

28. Manson, J. E., Bassuk, S. S., Buring, J. E. \& Group, V. R. Principal results of the VITamin D and OmegA-3 TriaL (VITAL) and updated meta-analyses of relevant vitamin D trials. J. Steroid Biochem. Mol. Biol. 198, 105522 (2020)

29. Bjelakovic, G. et al. Vitamin D supplementation for prevention of cancer in adults. Cochrane Database Syst. Rev. 6, CD007469 (2014).

30. Ong J. S. et al. Association of vitamin D levels and risk of ovarian cancer: a Mendelian randomization study. Int. J. Epidemiol. 45, 1619-1630 (2016).

31. Dimitrakopoulou, V. I. et al. Circulating vitamin D concentration and risk of seven cancers:
Mendelian randomisation study. BMJ 359, j4761 (2017).

32. Chandler, P. D. et al. Association between vitamin D genetic risk score and cancer risk in a large cohort of U.S. women. Nutrients 10, 55 (2018).

33. He, Y. et al. Exploring causality in the association between circulating 25-hydroxyvitamin D and colorectal cancer risk: a large Mendelian randomisation study. BMC Med. 16, 142 (2018)

34. Dong, J. et al. No association between vitamin $D$ status and risk of Barrett's esophagus or esophageal adenocarcinoma: a Mendelian randomization study. Clin. Gastroenterol. Hepatol. 17, 2227-2235.e1 (2019).

35. Winslow, U. C., Nordestgaard, B. G. \& Afzal, S. High plasma 25 -hydroxyvitamin $D$ and high risk of nonmelanoma skin cancer: a Mendelian randomization study of 97849 individuals. Br. J. Dermatol. 178, 1388-1395 (2018)

36. Chowdhury, R. et al. Vitamin D and risk of cause specific death: systematic review and meta-analysis of observational cohort and randomised intervention studies. BMJ 348, g1903 (2014).

37. Scragg, R. et al. Effect of monthly high-dose vitamin D supplementation on cardiovascular disease in the Vitamin D Assessment Study: a randomized clinical trial. JAMA Cardiol 2, 608-616 (2017)

38. Barbarawi, M. et al. Vitamin D supplementation and cardiovascular disease risks in more than 83000 individuals in 21 randomized clinical trials: a meta-analysis. JAMA Cardiol. 4, 765-776 (2019).

39. Afzal, S., Brondum-Jacobsen, P., Bojesen, S. E. \& Nordestgaard, B. G. Genetically low vitamin D concentrations and increased mortality: Mendelian randomisation analysis in three large cohorts. $B M J$ 349, g6330 (2014)

40. Manousaki, D. Mokry L. E., Ross, S., Goltzman, D. $\&$ Richards, J. B. Mendelian randomization studies do not support a role for vitamin D in coronary artery disease. Circ. Cardiovasc. Genet. 9, 349-356 (2016)

41. Larsson, S. C. et al. Serum 25-hydroxyvitamin D concentrations and ischemic stroke and its subtypes. Stroke 49, 2508-2511 (2018).

42. Huang, T. et al. Vitamin D and cause-specific vascular disease and mortality: a Mendelian randomisation study involving 99,012 Chinese and 106,911 European adults. BMC Med. 17, 160 (2019)

43. Revez, J. A. et al. Genome-wide association study identifies 143 loci associated with 25 hydroxyvitamin D concentration. Nat. Commun. 11, 1647 (2020)

44. Sluyter, J. D. et al. Effect of monthly, high-dose, long-term vitamin D supplementation on central blood pressure parameters: a randomized controlled trial substudy. J. Am. Heart Assoc. 6, e006802 (2017).

45. Vimaleswaran, K. S. et al. Association of vitamin D status with arterial blood pressure and hypertension risk: a Mendelian randomisation study. Lancet Diabetes Endocrinol. 2, 719-729 (2014).

46. Meng, X. et al. Phenome-wide Mendelian-randomization study of genetically determined vitamin D on multiple health outcomes using the UK Biobank study. Int. J. Epidemiol. 48 1425-1434 (2019).

47. Kwak, S. Y., Cho, Y., Oh, H. \& Shin, M. J. Association of circulating 25-hydroxyvitamin D levels with hypertension and blood pressure values in Korean adults: a Mendelian randomization study on a subset of the Korea National Health and Nutrition Survey 2011-2012 population. Nutr. Res. Pract. 13 498-508 (2019)

48. Chen, C. et al. Association of 25-hydroxyvitamin D with cardiometabolic risk factors and metabolic syndrome: a Mendelian randomization study. Nutr. J. 18, 61 (2019)

49. Boonen, $\mathrm{S}$. et al. Need for additional calcium to reduce the risk of hip fracture with vitamin $D$ supplementation: evidence from a comparative metaanalysis of randomized controlled trials. J. Clin Endocrinol. Metab. 92, 1415-1423 (2007)

50. Bolland, M. J., Grey, A., Gamble, G. D. \& Reid, I. R. The effect of vitamin D supplementation on skeletal, vascular, or cancer outcomes: a trial sequential meta-analysis. Lancet Diabetes Endocrinol. 2 307-320 (2014)

51. Avenell, A., Mak, J. C. \& O'Connell, D. Vitamin D and vitamin $D$ analogues for preventing fractures in post-menopausal women and older men. Cochrane Database Syst. Rev. 4, CD000227 (2014).

52. Yao, P. et al. Vitamin $D$ and calcium for the prevention of fracture: a systematic review and meta-analysis. JAMA Netw. Open 2, e 1917789 (2019).
53. Chakhtoura, M., Chamoun, N., Rahme, M. $\&$ Fuleihan, G. E. Impact of vitamin D supplementation on falls and fractures-a critical appraisal of the quality of the evidence and an overview of the available guidelines. Bone 131, 115112 (2020).

54. Bikle, D., Bouillon, R., Thadhani, R. \& Schoenmakers, I. Vitamin D metabolites in captivity? Should we measure free or total $25(\mathrm{OH}) \mathrm{D}$ to assess vitamin D status? J. Steroid Biochem. Mol. Biol. 173, 105-116 (2017).

55. LeBoff, M. S. et al. Effects of supplemental vitamin D on bone health outcomes in women and men in the VITamin D and OmegA-3 TriaL (VITAL). J. Bone Miner. Res. 35, 883-893 (2020)

56. Khaw, K. T. et al. Effect of monthly high-dose vitamin D supplementation on falls and non-vertebral fractures: secondary and post-hoc outcomes from the randomised, double-blind, placebo-controlled ViDA trial. Lancet Diabetes Endocrinol. 5, 438-447 (2017)

57. Reid, I. R. et al. Effect of monthly high-dose vitamin D on bone density in community-dwelling older adults substudy of a randomized controlled trial. J. Intern. Med. 282, 452-460 (2017).

58. Macdonald, H. M. et al. 25-hydroxyvitamin D threshold for the effects of vitamin D supplements on bone density: secondary analysis of a randomized controlled trial. J. Bone Miner. Res. 33, 1464-1469 (2018).

59. Hansen, K. E. et al. Treatment of vitamin D insufficiency in postmenopausal women: a randomized clinical trial. JAMA Intern. Med. 175, 1612-1621 (2015).

60. Aloia, J. et al. Vitamin D supplementation in elderly black women does not prevent bone loss: a randomized controlled trial. J. Bone Miner. Res. 33 , 1916-1922 (2018).

61. Rosendahl, J. et al. Effect of higher vs standard dosage of vitamin D3 supplementation on bone strength and infection in healthy infants: a randomized clinical trial. JAMA Pediatrics 172, 646-654 (2018).

62. Bouillon, R. Safety of high-dose vitamin D supplementation. J. Clin. Endocrinol. Metab. 105 1290-1291 (2020).

63. Larsson, S. C., Melhus, H. \& Michaelsson, K Circulating serum 25-hydroxyvitamin D levels and bone mineral density: Mendelian randomization study. J. Bone Miner. Res. 33, 840-844 (2018).

64. Sun, J. Y. et al. Circulating serum vitamin D levels and total body bone mineral density: a Mendelian randomization study. J. Cell Mol. Med. 23, 2268-2271 (2019).

65. Trajanoska, K. et al. Assessment of the genetic and clinical determinants of fracture risk: genome wide association and Mendelian randomisation study. $B M$ 362, k3225 (2018).

66. Kampe, A. et al. Genetic variation in GC and CYP2R1 affects 25-hydroxyvitamin D concentration and skeletal parameters: a genome-wide association study in 24-month-old Finnish children. PLoS Genet. 15, e 1008530 (2019)

67. Li, S. S. et al. Genetically low vitamin D Levels, bone mineral density, and bone metabolism markers: a Mendelian randomisation study. Sci. Rep. 6, 33202 (2016)

68. Beaudart, C. et al. The effects of vitamin D on skeletal muscle strength, muscle mass, and muscle power: a systematic review and meta-analysis of randomized controlled trials. J. Clin. Endocrinol. Metab. 99 4336-4345 (2014).

69. Tabrizi, R. et al. The effects of vitamin D supplementation on muscle function among postmenopausal women: a systematic review and meta-analysis of randomized controlled trials. EXCLI J. 18, 591-603 (2019).

70. Girgis, C. M., Clifton-Bligh, R. J., Hamrick, M. W., Holick, M. F. \& Gunton, J. E. The roles of vitamin D in skeletal muscle: form, function, and metabolism Endocr. Rev. 34, 33-83 (2013).

71. Sanders, K. M. et al. Annual high-dose oral vitamin D and falls and fractures in older women: a randomized controlled trial. JAMA 303, 1815-1822 (2010).

72. Bischoff-Ferrari, H. A. et al. Monthly high-dose vitamin $\mathrm{D}$ treatment for the prevention of functional decline: a randomized clinical trial. JAMA Intern. Med. 176, 175-183 (2016).

73. Gallagher, J. C. Vitamin D and falls-the dosage conundrum. Nat. Rev. Endocrinol. 12, 680-684 (2016).

74. Smith, L. M., Gallagher, J. C. \& Suiter, C. Medium doses of daily vitamin $D$ decrease falls and higher doses of daily vitamin D3 increase falls: a randomized clinical trial. J. Steroid Biochem. Mol. Biol. 173, 317-322 (2017). 
75. Michos, E. D. et al. Rationale and design of the Study To Understand Fall Reduction and Vitamin D in You (STURDY): a randomized clinical trial of vitamin D supplement doses for the prevention of falls in older adults. Contemp. Clin. trials 73, 111-122 (2018).

76. LeBoff, M. S et al. VITamin D and OmegA-3 TriaL (VITAL): effects of vitamin D supplements on risk of falls in the US population. J. Clin. Endocrinol. Metab. 105, 2929-2938 (2020)

77. Cashman, K. D., Ritz, C., Kiely, M. \& Odin, C. Improved dietary guidelines for vitamin D: application of individual participant data (IPD)-level meta-regression analyses. Nutrients 9, 469 (2017)

78. Bouillon, R. Vitamin D status in Africa is worse than in other continents. Lancet Glob. Health 8, e20-e2 (2020).

79. Bischoff-Ferrari, H. A. et al. Effect of vitamin D on falls: a meta-analysis. JAMA 291, 1999-2006 (2004).

80. Lange, N. E., Sparrow, D., Vokonas, P. \& Litonjua, A. A Vitamin $D$ deficiency, smoking, and lung function in the Normative Aging Study. Am. J. Respir. Crit. Care Med. 186, 616-621 (2012).

81. Janssens, $W$. et al. Vitamin D deficiency is highly prevalent in COPD and correlates with variants in the vitamin D-binding gene. Thorax 65, 215-220 (2010).

82. Maes, K., Serre, J., Mathyssen, C., Janssens, W. \& Gayan-Ramirez, G. Targeting vitamin D deficiency to limit exacerbations in respiratory diseases: utopia or strategy with potential? Calcif. Tissue Int. 106, 76-87 (2020)

83. Martineau, A. R. et al. Vitamin D supplementation to prevent acute respiratory infections: individual participant data meta-analysis. Health Technol. Assess. 23, 1-44 (2019).

84. Camargo, C. A. et al. Effect of monthly high-dose vitamin D supplementation on acute respiratory infections in older adults: a randomized controlled trial. Clin. Infect. Dis. 71, 311-317 (2019).

85. Chen, F. Y., Xiao, M., Ling, B., Liu, L. \& Chen, L. Vitamin $D$ does not improve lung function decline in COPD: a meta-analysis. Eur. Rev. Med. Pharmacol. Sci. 23, 8637-8644 (2019).

86. Sluyter, J. D. et al. Effect of monthly, high-dose, long-term vitamin $\mathrm{D}$ on lung function: a randomized controlled trial. Nutrients 9, 1353 (2017).

87. Bassatne, A. et al. The link between COVID-19 and vitamin D (VIVID): a systematic review and meta-analysis. Metabolism 119, 15475 (2021)

88. Murai, I. H. et al. Effect of a single high dose of vitamin D3 on hospital length of stay in patients with moderate to severe COVID-19: a randomized clinical trial. JAMA 325, 1053-1060 (2021).

89. Entrenas Castillo, M. et al. Effect of calcifediol treatment and best available therapy versus best available therapy on intensive care unit admission and mortality among patients hospitalized for COVID-19. a pilot randomized clinical study. J. Steroid Biochem Mol. Biol. 203, 105751 (2020)

90. Amin, H. \& Drenos, F. No evidence that vitamin D is able to prevent or affect the severity of COVID-19 in individuals with European ancestry: a Mendelian randomisation study of open data. BMJ Nutr. Prev. Health 4, 42-48 (2021).

91. Butler-Laporte, G. et al. Vitamin D and COVID-19 susceptibility and severity in the COVID-19 Host Genetics Initiative: a Mendelian randomization study. PLoS Med. 18, e1003605 (2021)

92. Li, W. et al. Vitamin D supplementation during pregnancy and the risk of wheezing in offspring a systematic review and dose-response meta-analysis. J. Asthma 56, 1266-1273 (2019).
93. Litonjua, A. A. et al. Six-year follow-up of a trial of antenatal vitamin D for asthma reduction. N. Engl. J. Med. 382, 525-533 (2020).

94. Manousaki, D. et al. Vitamin D levels and susceptibility to asthma, elevated immunoglobulin E levels, and atopic dermatitis: a Mendelian randomization study. PLoS Med. 14, e1002294 (2017).

95. Murdaca, G. et al. Emerging role of vitamin D in autoimmune diseases: an update on evidence and therapeutic implications. Autoimmun. Rev. 18, 102350 (2019).

96. Mokry, L. E. et al. Vitamin D and risk of multiple sclerosis: a mendelian randomization study. PLoS Med. 12, e1001866 (2015).

97. Jacobs, B. M., Noyce, A. J., Giovannoni, G. \& Dobson, R. BMI and low vitamin D are causal factors for multiple sclerosis: a Mendelian randomization study. Neurol. Neuroimmunol. Neuroinflamm 7, e662 (2020).

98. Gianfrancesco, M. A. et al. Evidence for a causal relationship between low vitamin $\mathrm{D}$, high $\mathrm{BMI}$, and pediatric-onset MS. Neurology 88, 1623-1629 (2017).

99. Rhead, B. et al. Mendelian randomization shows a causal effect of low vitamin D on multiple sclerosis risk. Neurol. Genet. 2, e97 (2016).

100. Bae, S. C. \& Lee, Y. H. Vitamin D level and risk of systemic lupus erythematosus and rheumatoid arthritis: a Mendelian randomization. Clin. Rheumatol. 37, 2415-2421 (2018)

101. Lund-Nielsen, J. et al. Vitamin D and inflammatory bowel disease: Mendelian randomization analyses in the Copenhagen studies and UK biobank. J. Clin. Endocrinol. Metab. 103, 3267-3277 (2018).

102. Manousaki, D. et al. Vitamin D levels and risk of type 1 diabetes: a Mendelian randomization study. PLoS Med. 18, e1003536 (2021).

103. Palacios, C., De-Regil, L. M., Lombardo, L. K. $\&$ Pena-Rosas, J. P. Vitamin D supplementation during pregnancy: updated meta-analysis on maternal outcomes. J. Steroid Biochem. Mol. Biol. 164, 148-155 (2016)

104. Palacios, C., Kostiuk, L. K. \& Pena-Rosas, J. P. Vitamin D supplementation for women during pregnancy. Cochrane Database Syst. Rev. 7, CD008873 (2019).

105. Roth, D. E. et al. Vitamin D supplementation in pregnancy and lactation and infant growth. N. Engl. J. Med. 379, 535-546 (2018).

106. Magnus, M. C. et al. Vitamin D and risk of pregnancy related hypertensive disorders: Mendelian randomisation study. BMJ 361, k2167 (2018).

107. Amrein, K. et al. Effect of high-dose vitamin D3 on hospital length of stay in critically ill patients with vitamin D deficiency: the VITdAL-ICU randomized clinical trial. JAMA 312, 1520-1530 (2014).

108. National Heart, L. et al. Early high-dose vitamin D3 for critically ill, vitamin D-deficient patients. N. Engl. J. Med. 381, 2529-2540 (2019).

109. Wactawski-Wende, J. et al. Calcium plus vitamin D supplementation and the risk of colorectal cancer N. Engl. J. Med. 354, 684-696 (2006).

110. Smith, H. et al. Effect of annual intramuscular vitamin D on fracture risk in elderly men and women-a population-based, randomized, double-blind, placebo-controlled trial. Rheumatology 46, 1852-1857 (2007).

111. Billington, $E$. O et al. Effect of high-dose vitamin D supplementation on peripheral arterial calcification: secondary analysis of a randomized controlled trial. Osteoporos. Int. 31, 2141-2150 (2020).

112. Durazo-Arvizu, R. A. et al. The reverse J-shaped association between serum total 25-Hydroxyvitamin D concentration and all-cause mortality: the impact of assay standardization. Am. J. Epidemiol. 185, 720-726 (2017)

113. Gaksch, M. et al. Vitamin D and mortality: individual participant data meta-analysis of standardized 25-hydroxyvitamin D in 26916 individuals from a European consortium. PLOS ONE 12, e0170791 (2017).

114. Mattila, T. et al. Airway obstruction, serum vitamin D and mortality in a 33-year follow-up study. Eur. J. Clin. Nutr. 73, 1024-1032 (2019).

115. Zhang, Y. et al. Association between vitamin D supplementation and mortality: systematic review and meta-analysis. BMJ 366, 14673 (2019).

116. Ordonez-Mena, J. M. et al. Genetic variants in the vitamin D pathway, 25(OH)D levels, and mortality in a large population-based cohort study. J. Clin. Endocrinol. Metab. 102, 470-477 (2016).

117. Aspelund, T. et al. Effect of genetically low 25-hydroxyvitamin D on mortality risk: Mendelian randomization analysis in 3 large European cohorts. Nutrients 11, 74 (2019).

118. Ong, J. S. et al. Vitamin D and overall cancer risk and cancer mortality: a mendelian randomization study. Hum. Mol. Genet. 27, 4315-4322 (2018).

119. Bouillon, R. \& Bikle, D. Vitamin D metabolism revised fall of dogmas. J. Bone Miner. Res. 34, 1985-1992 (2019).

120. Aatsinki, S. M. et al. Fasting-induced transcription factors repress vitamin $D$ bioactivation, a mechanism for vitamin D deficiency in diabetes. Diabetes $\mathbf{6 8}$, 918-931 (2019)

121. Roizen, J. D. et al. Obesity decreases hepatic 25-hydroxylase activity causing low serum 25-hydroxyvitamin D. J. Bone Miner. Res. 34, 1068-1073 (2019).

122. Atkinson, S. A. Recommendations on vitamin D needs in multiple sclerosis from the MS Society of Canada. Public Health Nutr. 23, 1278-1279 (2020).

123. Wang, T. J. et al. Common genetic determinants of vitamin $D$ insufficiency: a genome-wide association study. Lancet 376, 180-188 (2010).

124. Wu, H. X. et al. Effects of vitamin D supplementation on the outcomes of patients with pulmonary tuberculosis: a systematic review and meta-analysis. BMC Pulm. Med. 18, 108 (2018).

\section{Author contributions}

R.B., D.M. and K.T. researched data for the article. All authors contributed substantially to discussion of the content. R.B. and D.M. wrote the article. All authors reviewed and/or edited the manuscript before submission.

\section{Competing interests}

R.B. received modest speaking or consultancy fees from Fresenius, Abiogen, FAES Farma, Ceres and Proctor and Gamble. J.B.R. has worked as a consultant to GlaxoSmithKline and Deerfield Capital. The other authors declare no competing interests

\section{Peer review information}

Nature Reviews Endocrinology thanks D. Bikle, C. Carlberg,

$\mathrm{G}$. Jones and the other, anonymous, reviewer(s) for their contribution to the peer review of this work.

\section{Publisher's note}

Springer Nature remains neutral with regard to jurisdictional claims in published maps and institutional affiliations.

\section{Supplementary information}

The online version contains supplementary material available at https://doi.org/10.1038/s41574-021-00593-z.

(c) Springer Nature Limited 2021 